\title{
Does Parametric fMRI Analysis with SPM Yield Valid Results? - An Empirical Study of 1484 Rest Datasets
}

Anders Eklund, Mats Andersson, Camilla Josephson,

Magnus Johannesson and Hans Knutsson

\section{Linköping University Post Print}

N.B.: When citing this work, cite the original article.

Original Publication:

Anders Eklund, Mats Andersson, Camilla Josephson, Magnus Johannesson and Hans Knutsson, Does Parametric fMRI Analysis with SPM Yield Valid Results? - An Empirical Study of 1484 Rest Datasets, 2012, NeuroImage.

http://dx.doi.org/10.1016/j.neuroimage.2012.03.093

Copyright: Elsevier

http://www.elsevier.com/

Postprint available at: Linköping University Electronic Press

http://urn.kb.se/resolve?urn=urn:nbn:se:liu:diva-76118 


\title{
Does Parametric fMRI Analysis with SPM Yield Valid Results?
}

\author{
An Empirical Study of 1484 Rest Datasets
}

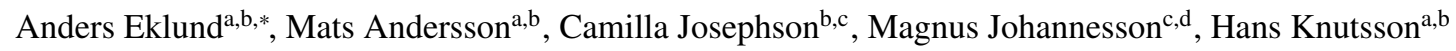 \\ ${ }^{a}$ Division of Medical Informatics, Department of Biomedical Engineering, Linköping University, Linköping, Sweden \\ ${ }^{b}$ Center for Medical Image Science and Visualization (CMIV), Linköping University, Linköping, Sweden \\ ${ }^{c}$ Department of Management and Engineering, Linköping University, Linköping, Sweden \\ ${ }^{d}$ Department of Economics, Stockholm School of Economics, Stockholm, Sweden
}

\begin{abstract}
The validity of parametric functional magnetic resonance imaging (fMRI) analysis has only been reported for simulated data. Recent advances in computer science and data sharing make it possible to analyze large amounts of real fMRI data. In this study, 1484 rest datasets have been analyzed in SPM8, to estimate true familywise error rates. For a familywise significance threshold of $5 \%$, significant activity was found in $1 \%-70 \%$ of the 1484 rest datasets, depending on repetition time, paradigm and parameter settings. This means that parametric significance thresholds in SPM both can be conservative or very liberal. The main reason for the high familywise error rates seems to be that the global AR(1) auto correlation correction in SPM fails to model the spectra of the residuals, especially for short repetition times. The findings that are reported in this study cannot be generalized to parametric fMRI analysis in general, other software packages may give different results. By using the computational power of the graphics processing unit (GPU), the 1484 rest datasets were also analyzed with a random permutation test. Significant activity was then found in $1 \%-19 \%$ of the datasets. These findings speak to the need for a better model of temporal correlations in fMRI timeseries.
\end{abstract}

Keywords:

Functional magnetic resonance imaging (fMRI), Familywise error rate, Random field theory, Non-parametric statistics, Random permutation test, Graphics processing unit (GPU)

\section{Introduction}

It has been debated for a long time if the assumptions that are required for standard parametric approaches really are appropriate for functional magnetic resonance imaging (fMRI) data. It has also been debated how the problem of multiple testing should be solved. This debate gained new momentum when significant brain activity was found in a dead salmon (Bennett et al., 2010). The recent advances in computer science, e.g. graphics processing units (GPUs), make it possible to perform conventional fMRI analysis in a few seconds (Eklund et al., 2011a, 2012). This permits using thousands of studies in the evaluation of analysis and inference procedures in fMRI data analysis, which was not previously possible. In this study, a large number of rest datasets have been analyzed to show that temporal correlations in resting state fMRI timeseries may show a more complicated structure, than previously assumed in conventional statistical models. Specifically, the autoregression models used by SPM are shown to fail to accommodate a preponderance of low frequencies in resting fMRI timeseries. The

${ }^{*}$ Corresponding author at: Division of Medical Informatics, Department of Biomedical Engineering, Linköping University, University Hospital, 58185 Linköping, Sweden, Tel: +46 (0)13 - 2867 25, Fax: +46 (0)13 - 101902

Email address: anders .eklund@liu.se (Anders Eklund) result of this is familywise error rates that are higher than the expected ones, especially for short repetition times.

There have been some studies that show that parametric significance thresholds from random field theory are conservative, mainly for multi subject fMRI (Poline et al., 1997; Nichols and Holmes, 2001; Nichols and Hayasaka, 2003; Hayasaka and Nichols, 2003a) but also for single subject fMRI (Friston et al., 1994; Hayasaka and Nichols, 2003b). These studies are mainly based on simulated data, which never can capture all properties of real data. The only study that used real data to estimate familywise error rates (Zarahn et al., 1997) merely used 17 datasets, which is inadequate for a good estimate.

The idea of the empirical study is to analyze a large number of rest (Null) datasets and simply count the number of datasets with significant activity. If a familywise significance threshold of $5 \%$ is used, activity should be found in 5 out of 100 rest datasets. The empirical study is thus a way to investigate if the assumptions about the null distribution hold. An important note is that the null hypothesis does not state that there is not any brain activity in rest data (there is always activity in the brain), but that the rest data do not contain any brain activity that is correlated with a randomly selected regressor. The main difficulty of doing such a study is how to get hold of a large number of rest datasets. 


\section{Data}

Resting state fMRI data is commonly collected to study functional connectivity (Biswal et al., 1995). As the aim of this study is to investigate Null distributions, resting state fMRI data is what we need. For these reasons, the freely available resting state fMRI datasets in the Neuroimaging Informatics Tools and Resources Clearinghouse (NITRC) 1000 functional connectomes project (Biswal et al., 2010) have been used. The data is fully anonymized and is released under a license that allows unrestricted non-commercial use, researchers are free to publish any portion of the data set. The enthusiastic researcher can thus repeat the study by using the same data. More information about the project can be found at

http://fcon_1000.projects.nitrc.org/ .

A total of 1484 resting state fMRI datasets were downloaded from the website, see Table 1 for more information about the datasets, requiring about $85 \mathrm{~GB}$ of storage. For each subject there is also a high resolution anatomical volume. The number of subjects is not 1484 , but 1253 . For the New Haven data there are 2 or 4 rest datasets per subject and for the ICBM data there are 3 rest datasets per subject. We believe that these datasets are a good representation of different subjects, MR scanners and MR settings.

\section{Methods}

The 1484 rest datasets were analyzed in $\mathrm{SPM}^{1}$ (updated to version 4290), by using a Matlab batch script. We chose to use the SPM software as it, to our knowledge, is the most common software for fMRI analysis. The findings that are reported in this study cannot be generalized to parametric fMRI analysis in general, as other fMRI software packages (e.g. $\mathrm{FSL}^{2}, \mathrm{AFNI}^{3}$, fmristat ${ }^{4}$ ), for example, use other models of the auto correlation.

\subsection{Preprocessing}

Each dataset was first motion corrected and then seven amounts of smoothing (4, 6, 8, 10, 12, 14, 16 mm FWHM) were applied to the motion corrected volumes. Slice timing correction was not applied to the volumes since information about the slice order (i.e. continuous or interleaved) is not available.

The analysis was performed both with and without estimated motion parameters as additional regressors in the design matrix. The additional regressors will reduce the variance of the residuals, and can thereby increase the test values. At the same time, the residual energy at low frequencies can be reduced, as estimated motion parameters often are dominated by low frequencies. The spectrum of the residuals can thereby become

\footnotetext{
${ }^{1}$ http://www.fil.ion.ucl.ac.uk/spm/

${ }^{2}$ http://www.fmrib.ox.ac.uk/fsl/

${ }^{3}$ http://afni.nimh.nih.gov/afni/

${ }^{4}$ http://www.math.mcgill.ca/keith/fmristat/
}

more flat (white), resulting in a decrease of the test values. Motion regressors can also reduce spikes and jumps in the data, resulting in a better estimate of the non-sphericity.

The high resolution anatomical volume could have been used to segment the brain into gray and white matter, but in a number of cases the registration between the functional dataset and the anatomical dataset failed. The reason for this seems to be that the functional and the anatomical data are stored in different coordinate systems. Due to this, only the functional datasets were used in the analysis. As this study is about single subject fMRI analysis, the datasets were not warped into a standard brain space.

\subsection{Statistical analysis with SPM}

The statistical analysis was performed in eight different ways, four block based designs (B1, B2, B3, B4) and four event related designs (E1, E2, E3, E4) were used. The length of activity and rest periods are given in Table 2 . For data that conforms to Gaussian white noise, the choice of regressors does not matter. The significance threshold will always be the same, as white noise has the same energy for all frequencies and phases. This is, however, not necessarily true for resting state fMRI data. Two regressors were used for all designs, the stimulus paradigm convolved with the hemodynamic response function (canonical) and its temporal derivative.

Table 2: Length of activity and rest periods, for the block based (B) and the event related (E) designs, R stands for randomized.

\begin{tabular}{|c|c|c|}
\hline Paradigm & Activity periods (s) & Rest periods (s) \\
\hline B1 & 10 & 10 \\
B2 & 15 & 15 \\
B3 & 20 & 20 \\
B4 & 30 & 30 \\
E1 & 2 & 6 \\
E2 & 4 & 8 \\
E3 & $1-4(\mathrm{R})$ & $3-6(\mathrm{R})$ \\
E4 & $3-6(\mathrm{R})$ & $4-8(\mathrm{R})$ \\
\hline
\end{tabular}

A t-test value was calculated in each voxel, then a voxelwise as well as a cluster based threshold, for a familywise error rate of 5\%, was applied. For the cluster based threshold, the activity map was first thresholded at $\mathrm{p}=0.001$ (uncorrected). The size of the largest cluster was then compared to the random field theory cluster extent threshold (Friston et al., 1994). The number of datasets with significant activity was finally divided by the number of analyzed datasets, to obtain the familywise error rate.

\subsection{SPM settings}

Except for the small modification of adding time derivatives, the default SPM settings were used in all processing steps (e.g. global AR(1) auto correlation correction, high pass filtering with a cutoff period of 128 seconds). The variable

defaults.stats.fmri.ufp 
Table 1: Information about the rest datasets that have been used for the empirical study. For the New Haven data there are 2 or 4 rest datasets per subject, for the ICBM data there are 3 rest datasets per subject.

\begin{tabular}{|c|c|c|c|c|c|c|c|c|}
\hline Institution & Persons & \# Subjects & Ages & TR (s) & \# Time points & Voxel size (mm) & Volume resolution & Field strength (T) \\
\hline Ann Arbor & Monk, C.S., Seidler, R.D., Peltier, S.J. & 25 & $13-40$ & 1.0 & 295 & $3.44 \times 3.44 \times 3.00$ & $64 \times 64 \times 40$ & 3.0 \\
\hline Ann Arbor & Monk, C.S., Seidler, R.D., Peltier, S.J. & 36 & $19-80$ & 1.0 & 395 & $3.44 \times 3.44 \times 3.20$ & $64 \times 64 \times 16$ & 3.0 \\
\hline Atlanta & Mayberg, H.S. & 28 & $22-57$ & 2.0 & 205 & $3.44 \times 3.44 \times 4.00$ & $64 \times 64 \times 20$ & 3.0 \\
\hline Baltimore & Pekar, J.J., Mostofsky, S.H. & 23 & $20-40$ & 2.5 & 123 & $2.67 \times 2.67 \times 3.00$ & $96 \times 96 \times 47$ & 3.0 \\
\hline Bangor & Colcombe, S. & 20 & $19-38$ & 2.0 & 265 & $3.00 \times 3.00 \times 3.00$ & $80 \times 80 \times 34$ & 3.0 \\
\hline Beijing & Zang, Y.F. & 198 & $18-26$ & 2.0 & 225 & $3.13 \times 3.13 \times 3.60$ & $64 \times 64 \times 33$ & 3.0 \\
\hline Berlin & Margulies, D. & 26 & $23-44$ & 2.3 & 195 & $3.00 \times 3.00 \times 4.00$ & $64 \times 64 \times 34$ & 3.0 \\
\hline Cambridge & Buckner, R.L. & 198 & $18-30$ & 3.0 & 119 & $3.00 \times 3.00 \times 3.00$ & $72 \times 72 \times 47$ & 3.0 \\
\hline Cleveland & Lowe, M.J. & 31 & $24-60$ & 2.8 & 127 & $2.00 \times 2.00 \times 4.00$ & $128 \times 128 \times 31$ & 3.0 \\
\hline ICBM & Evans, A.C. & 86 & $19-85$ & 2.0 & 128 & $4.00 \times 4.00 \times 5.50$ & $64 \times 64 \times 23$ & 3.0 \\
\hline Leiden & Rombouts, S.A.R.B. & 12 & $20-27$ & 2.2 & 215 & $3.44 \times 3.44 \times 3.44$ & $64 \times 64 \times 38$ & 3.0 \\
\hline Leiden & Rombouts, S.A.R.B. & 19 & $18-28$ & 2.2 & 215 & $3.44 \times 3.44 \times 3.44$ & $64 \times 64 \times 38$ & 3.0 \\
\hline Leipzig & Villringer, A. & 37 & $20-42$ & 2.3 & 195 & $3.00 \times 3.00 \times 4.00$ & $64 \times 64 \times 34$ & $\mathrm{n} / \mathrm{a}$ \\
\hline Milwaukee & $\mathrm{Li}$, S.J. & 18 & $\mathrm{n} / \mathrm{a}$ & 2.0 & 175 & $3.75 \times 3.75 \times 6.00$ & $64 \times 64 \times 20$ & 3.0 \\
\hline Milwaukee & Li, S.J. & 46 & $44-65$ & 2.0 & 175 & $4.00 \times 3.75 \times 3.75$ & $64 \times 64 \times 36$ & 3.0 \\
\hline Munchen & Sorg, C., Riedl, V. & 16 & $63-73$ & 3.0 & 72 & $3.13 \times 3.13 \times 4.40$ & $64 \times 64 \times 33$ & 1.5 \\
\hline Newark & Biswal, B. & 19 & $21-39$ & 2.0 & 135 & $3.44 \times 3.44 \times 5.00$ & $64 \times 64 \times 32$ & 3.0 \\
\hline New Haven & Hampson, M. & 19 & $18-48$ & 1.0 & 249 & $3.44 \times 3.44 \times 6.00$ & $64 \times 64 \times 16$ & 3.0 \\
\hline New Haven & Hampson, $\mathrm{M}$. & 16 & $18-42$ & 1.5 & 181 & $3.44 \times 3.44 \times 5.50$ & $64 \times 64 \times 22$ & 3.0 \\
\hline New York & Milham, M.P., Castellanos, F.X. & 25 & $20-50$ & 2.0 & 192 & $3.00 \times 3.00 \times 3.00$ & $64 \times 64 \times 39$ & 3.0 \\
\hline New York & Milham, M.P., Castellanos, F.X. & 20 & $18-46$ & 2.0 & 175 & $3.00 \times 3.00 \times 4.00$ & $64 \times 80 \times 33$ & 3.0 \\
\hline Orangeburg & Hoptman, M. & 20 & $20-55$ & 2.0 & 165 & $3.50 \times 3.50 \times 5.00$ & $64 \times 64 \times 22$ & 1.5 \\
\hline Oulu & Kiviniemi, V.J., Veijiola, J. & 103 & $20-23$ & 1.8 & 245 & $4.00 \times 4.00 \times 4.40$ & $64 \times 64 \times 28$ & 1.5 \\
\hline Oxford & Smith, S.M., Mackay, C. & 22 & $20-35$ & 2.0 & 175 & $3.00 \times 3.00 \times 3.50$ & $64 \times 64 \times 34$ & 3.0 \\
\hline Palo Alto & Greicius, $\mathrm{M}$. & 17 & $22-46$ & 2.0 & 235 & $3.44 \times 3.44 \times 4.90$ & $64 \times 64 \times 29$ & $\mathrm{n} / \mathrm{a}$ \\
\hline Pittsburgh & Siegle, G. & 17 & $25-54$ & 1.5 & 275 & $3.13 \times 3.13 \times 3.20$ & $64 \times 64 \times 29$ & 3.0 \\
\hline Queensland & McMahon, K. & 19 & $20-34$ & 2.1 & 190 & $3.60 \times 3.60 \times 3.60$ & $64 \times 64 \times 36$ & 3.0 \\
\hline Saint Louis & Schlaggar, B., Petersen, S. & 31 & $21-29$ & 2.5 & 127 & $4.00 \times 4.00 \times 4.00$ & $64 \times 64 \times 32$ & 3.0 \\
\hline Taipei & Lin, C.P. & 14 & $\mathrm{n} / \mathrm{a}$ & 2.0 & 295 & $3.75 \times 3.75 \times 4.00$ & $64 \times 64 \times 32$ & 3.0 \\
\hline Taipei & Lin, C.P. & 8 & $\mathrm{n} / \mathrm{a}$ & 2.0 & 175 & $3.44 \times 3.44 \times 4.00$ & $64 \times 64 \times 33$ & 3.0 \\
\hline
\end{tabular}

was changed from 0.001 to 0.05 , to make sure that the rest datasets pass the first overall F-test that is applied in SPM. Without this modification, the error message "please check your data, there are no significant voxels" will arrise for rather many of the analyses and then no further analysis will be performed.

Global normalisation is not always used for activation data but is commonly applied for resting state data. The analysis was therefore performed both with and without global normalization.

\subsection{Non-parametric analysis}

To compare SPM8 to a non-parametric approach (Eklund et al., 2011a), a random permutation test, with 10000 permutations, was also applied to each dataset. This was only done for $8 \mathrm{~mm}$ of smoothing, as it would take several months to repeat the random permutation test for all amounts of smoothing. The significance thresholds from the random permutation test can, however, not be directly compared to the ones from SPM, as, for example, the motion correction is performed in a different way (Eklund et al., 2010). The random permutation test does not use global normalization or motion regressors.

The random permutation test applies whitening with a voxelwise $\mathrm{AR}(4)$ model prior to the permutations, since the permutation test requires the samples to be exchangeable under the null hypothesis. Prior to the whitening, a cubic detrending (Friman et al., 2004) is first applied to remove the mean and any polynomial trend up to the third order. The AR(4) coefficients are then independently estimated for each voxel timeseries, by solving the Yule-Walker equations. To improve the estimates, the coefficients are spatially smoothed with a Gaussian kernel of $8 \mathrm{~mm}$
FWHM. Our amount of smoothing is less than the amount (15 mm FWHM) proposed by (Worsley et al., 2002), but close to the optimal amount (6.5 - 7.5 mm FWHM) found by further investigation (Gautama and Hulle, 2004). Normalized convolution (Knutsson and Westin, 1993) is used to prevent that the smoothing is affected by voxels outside the brain. Normalized convolution produces a result that is weighted with a voxel-wise certainty, in our case the certainty is set to 1 for brain voxels and 0 otherwise. The result of our whitening is a flexible and spatially non-stationary model of temporal correlations.

\subsection{Power spectra}

To estimate power spectra of fMRI rest data, smoothed with a $8 \mathrm{~mm}$ kernel, standardized residual timeseries from SPM were Fourier transformed and average power spectra were calculated. Windowing is normally considered to be an important operation in signal processing, a window function is as a rule applied to the timeseries prior to the Fourier transform. This was not done in our case, as SPM does not apply a window function prior to the estimation of the AR model. To avoid conversion between different sampling rates, the study was separately done for three different repetition times, $1 \mathrm{~s}$ (97 datasets), $2 \mathrm{~s}$ (796 datasets) and $3 \mathrm{~s}$ (214 datasets). Two spectra were estimated for each repetition time, spectrum of the original residuals (auto correlation correction turned off in SPM) and the whitened residuals (auto correlation correction turned on in SPM). To give the reader an idea of the spectrum that is estimated by SPM, the ratio of the original and the residual spectra were calculated. Note that these ratio spectra are not actual estimates from SPM, they are 
only used to increase the understanding of SPM's whitening for different repetition times.

Power spectra were also calculated for the random permutation test, to see the result of the voxel-wise AR(4) whitening prior to the permutations. All the timeseries were, as standardized residuals from SPM8, normalized to have a variance of 1 .

\subsection{Which parameters affect the familywise error rate?}

The GLM framework is based on several assumptions about the residuals. One important assumption is that residuals are white (sphericity). This assumption is, for example, related to the repetition time and if motion regressors are used or not. The whitening that is used in SPM assumes that the temporal correlations of the residuals are stationary over voxels. This assumption could be tested indirectly, by changing the F-test threshold (defaults.stats.fmri.ufp) that determines which voxels that are used to estimate the non-sphericity. In our opinion, it is however clear that this assumption is violated. Estimated AR(1) parameters often yield a spatial pattern that is similar to the default mode network (Worsley et al., 2002). The whitening performance is not likely to be affected by the number of voxels that are used to estimate the non-sphericity, as long as the same whitening is applied to all timeseries.

Random field theory requires the activity map to be smooth, to be a good lattice approximation to random fields. This is related to the amount of smoothing that is applied to the volumes. The smoothing also affects the assumption that the residuals are normally distributed, as smoothing, by the central limit theorem, will make the data more Gaussian.

A good fMRI software should, for example, be invariant to the repetition time and the paradigm design and always give valid results. To determine the analysis parameters that have the greatest effect on the familywise error rate, we used a binary logistic regression analysis looking at the effects of smoothing, paradigm, repetition time, inference level and the application of motion regressors and global normalisation (see Table 3). We repeated a similar analysis for both the SPM8 results and the non-parametric results (omitting the smoothing, motion regressors and global normalisation parameters for the non-parametric results). The number of analyses (trials) and false positives (events) for each level combination were analyzed in Minitab. A significance level of 5\% was used to test the significance of each parameter.

To get independent measurements, it would be necessary to use different datasets for each level combination. The reported results are not corrected for dependence between the measurements, the significance of each parameter may therefore be overestimated.

\section{Results}

Two of the datasets (number 905 and 1310) were removed from the study, due to empty brain masks. For some of the 82992 analyses (1482 datasets $\times 7$ amounts of smoothing $\times$ 8 paradigms) the error message "please check your data, there are no significant voxels" appeared in the SPM software and no
Table 3: Parameters used in the binary logistic regression analysis, and their levels.

\begin{tabular}{|c|c|}
\hline Parameter & Levels \\
\hline Smoothing & Low $(4-8 \mathrm{~mm})$, High $(10-16 \mathrm{~mm})$ \\
Paradigm & Block, Event \\
Repetition time & $1,3 \mathrm{~s}$ \\
Inference level & Voxel level, Cluster level \\
Motion regressors & No, Yes \\
Global normalization & No, Yes \\
\hline
\end{tabular}

further analysis was performed. The number of occurances for different parameter settings are given in Table 4. The error message especially appeared for high amounts of smoothing. For these cases, the datasets were classified as inactive, i.e. counted as true negatives. The thresholds for these cases are therefore plotted as zeros.

Table 4: Number of error messages for different parameter settings, out of 82 992 analyses per parameter setting, $\mathrm{GN}=$ global normalization, $\mathrm{MR}=$ motion regressors.

\begin{tabular}{|c|c|}
\hline Parameter setting & Number of error messages \\
\hline No GN, no MR & 186 \\
No GN, MR & 0 \\
GN, no MR & 503 \\
GN, MR & 20 \\
\hline
\end{tabular}

\subsection{Familywise error rates and thresholds}

Familywise error rates for SPM8, without global normalization and motion regressors, are given in Figures 1(a) - 1(b). Familywise error rates for SPM8, with global normalization and motion regressors, are given in Figures 2(a) - 2(b). A parallel coordinate approach (Inselberg, 1985) was used to plot the familywise error rate as function of paradigm, smoothing and repetition time in a $2 \mathrm{D}$ plot. Familywise error rates for the random permutation test are given in Figures 3(a) - 3(b). The estimated familywise error rates follow a binomial distribution. Approximate $95 \%$ confidence intervals for a familywise error rate of $5 \%$, for different repetition times, are included in the figures. The confidence intervals are also given in Table 5.

Table 5: Approximate 95\% confidence intervals for a familywise error rate of $5 \%$, for different repetition times.

\begin{tabular}{|c|c|}
\hline Repetition time & 95\% Confidence interval \\
\hline 1 s (97 datasets) & $1.0 \%-9.0 \%$ \\
2 s (796 datasets) & $3.5 \%-6.5 \%$ \\
3 s (214 datasets) & $2.0 \%-8.0 \%$ \\
\hline
\end{tabular}

The maximum test values/cluster sizes, the random field theory significance thresholds and the random permutation test significance thresholds, for $8 \mathrm{~mm}$ smoothing, are given in Figures 4(a) - 7(d). The data for these plots were generated without global normalization and without motion regressors in the design matrix, as the random permutation test does not use these settings. 


\subsection{Power spectra}

The estimated power spectra, for SPM8 and the first block based design, are given in Figures 8(a) - 11(c). Similar results were obtained for the other designs. Power spectra for the voxel-wise $\mathrm{AR}(4)$ whitening prior to the permutation test are given in Figures 12(a) - 13(c).

\subsection{Regression analysis}

The result of the regression analysis is for SPM8 given in Table 6 and for the random permutation test given in Table 7. 
Voxel level inference, SPM8, no global normalization, no motion regressors

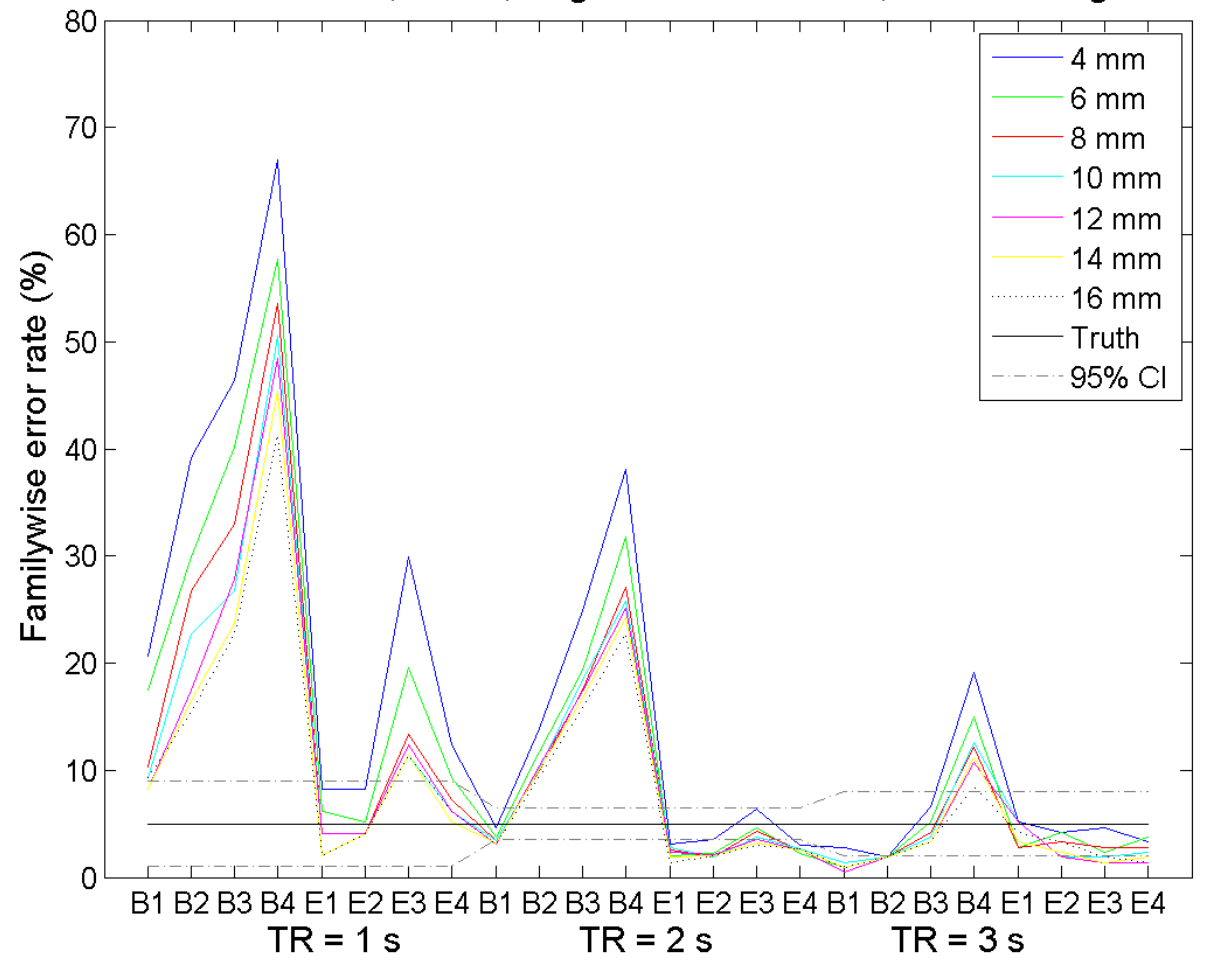

(a)

Cluster level inference, SPM8, no global normalization, no motion regressors

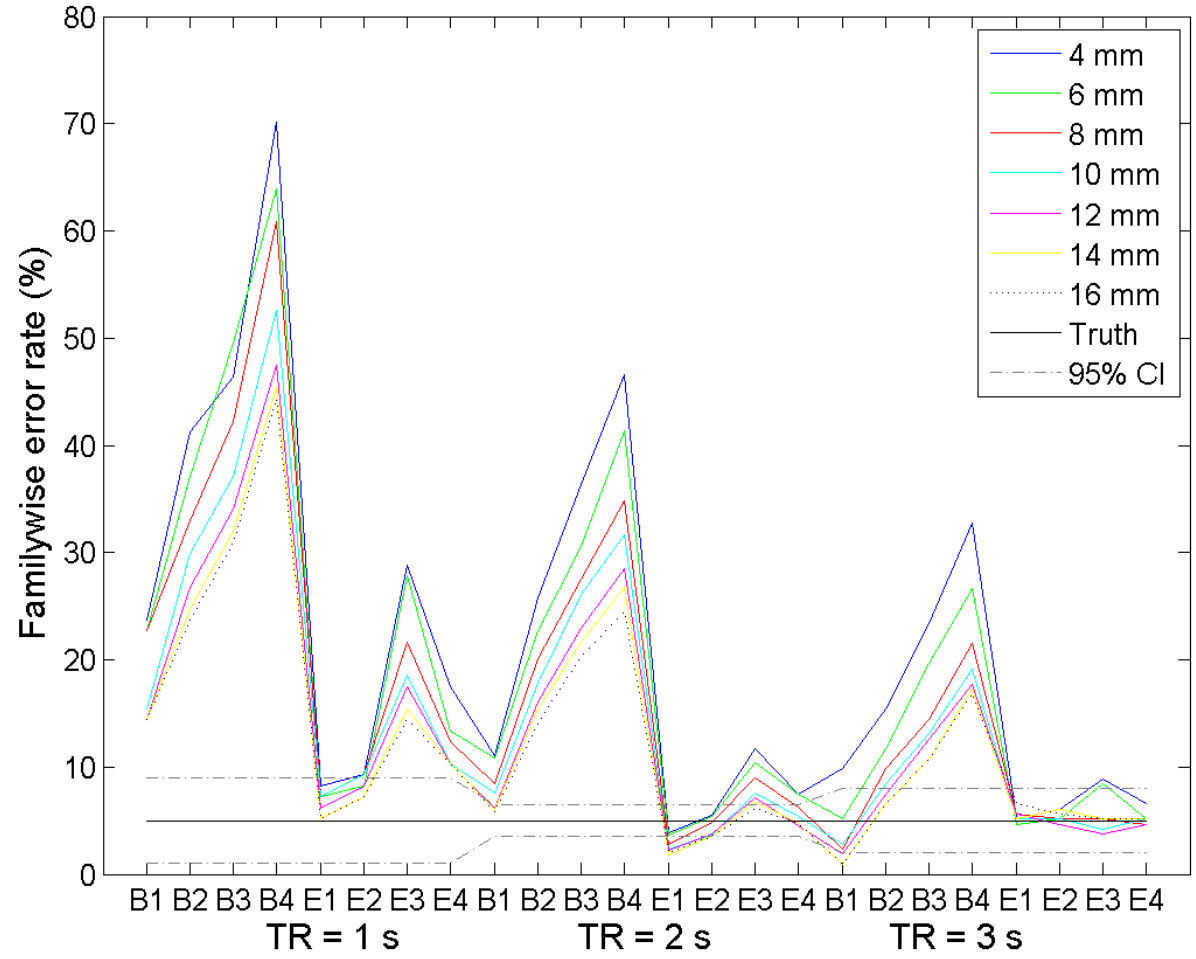

(b)

Figure 1: Familywise error rates for SPM8 as function of paradigm and repetition time, when global normalization was turned off and motion regressors not were included. The parallel lines represent different amounts of smoothing (4-16 mm), more smoothing yields lower familywise error rates. The true familywise error rate of $5 \%$ and the $95 \%$ confidence intervals are included as reference. 


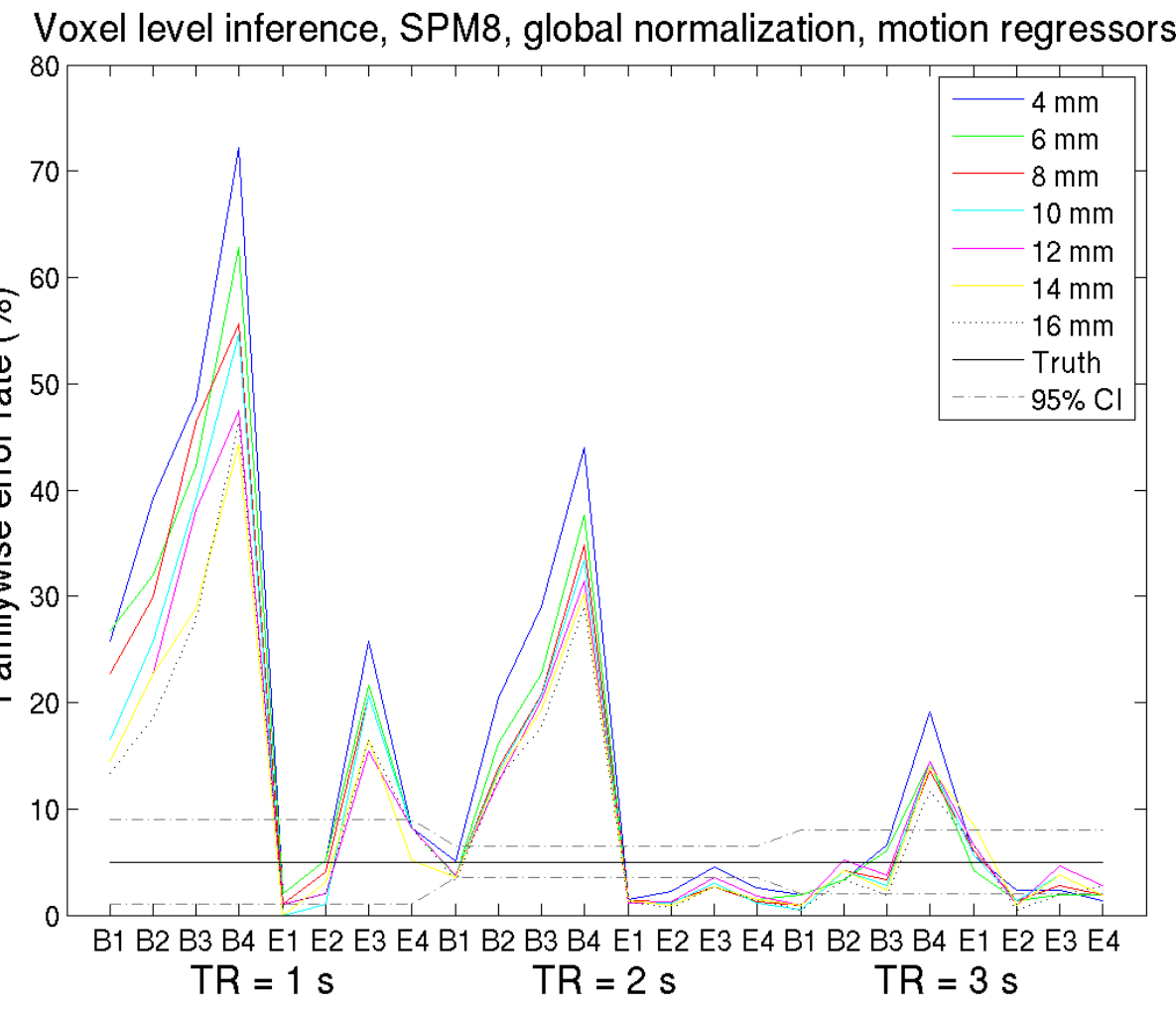

(a)

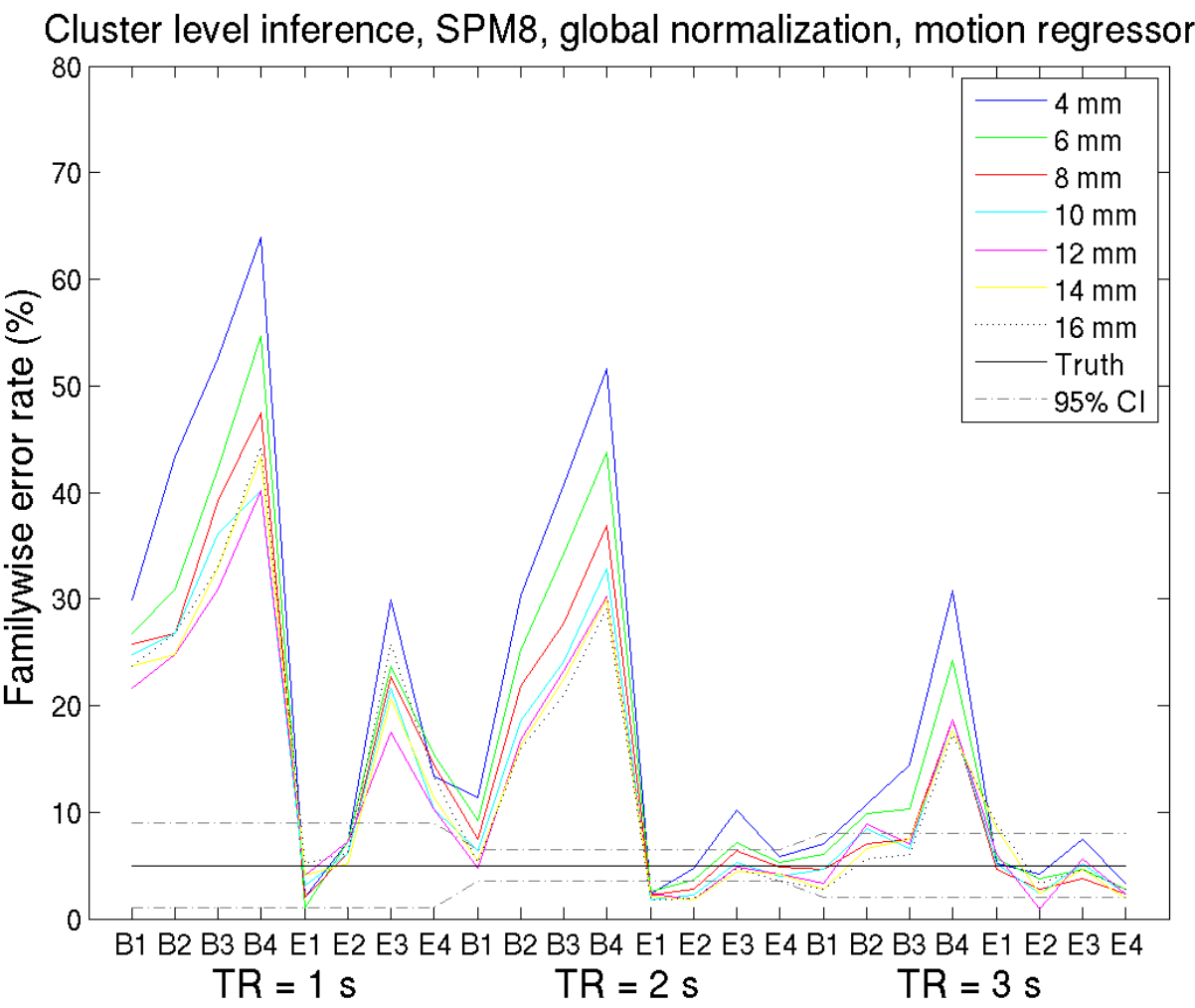

(b)

Figure 2: Familywise error rates for SPM8 as function of paradigm and repetition time, when global normalization was turned on and motion regressors were included. The parallel lines represent different amounts of smoothing (4 - $16 \mathrm{~mm})$, more smoothing yields lower familywise error rates. The true familywise error rate of $5 \%$ and the $95 \%$ confidence intervals are included as reference. 


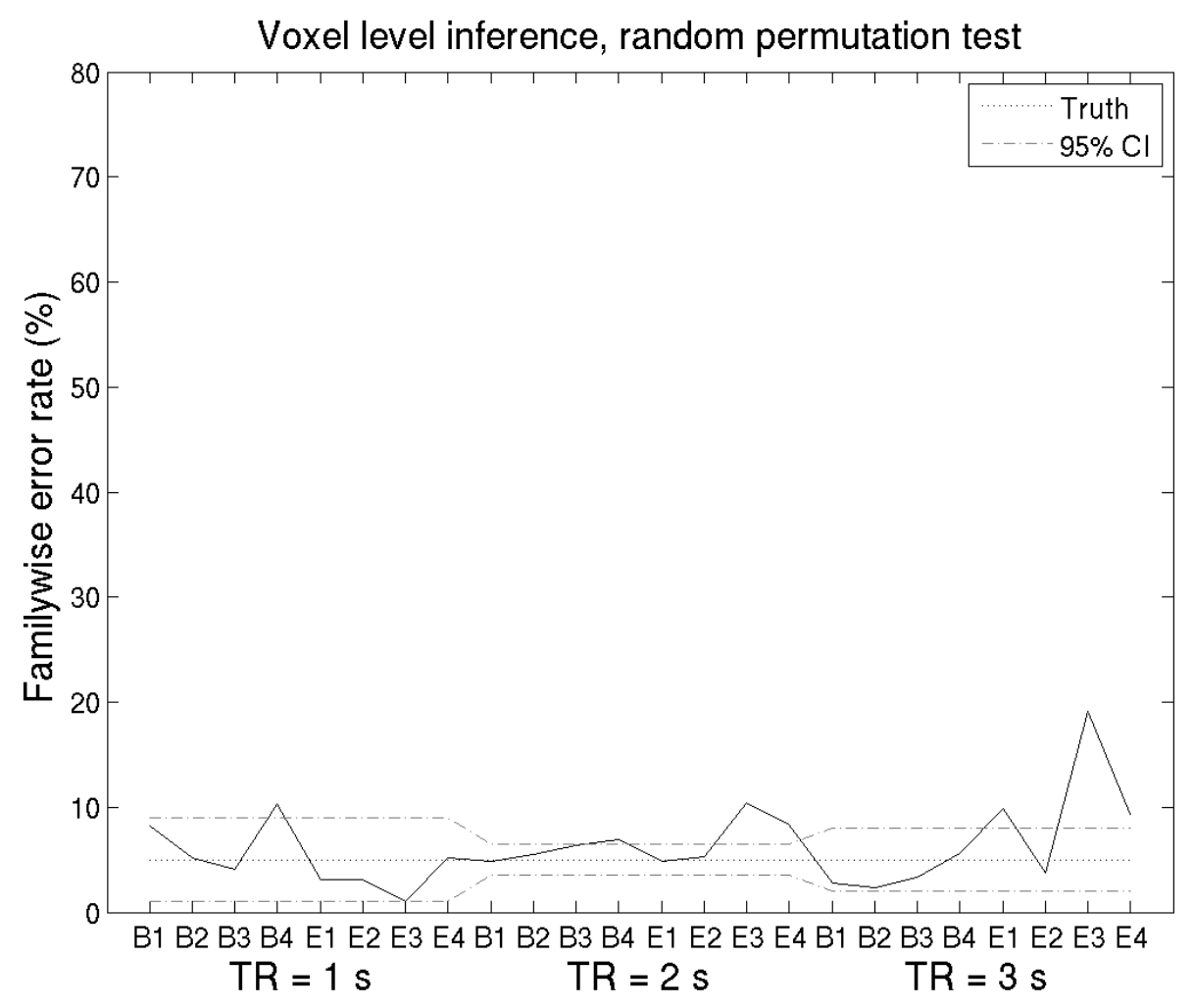

(a)

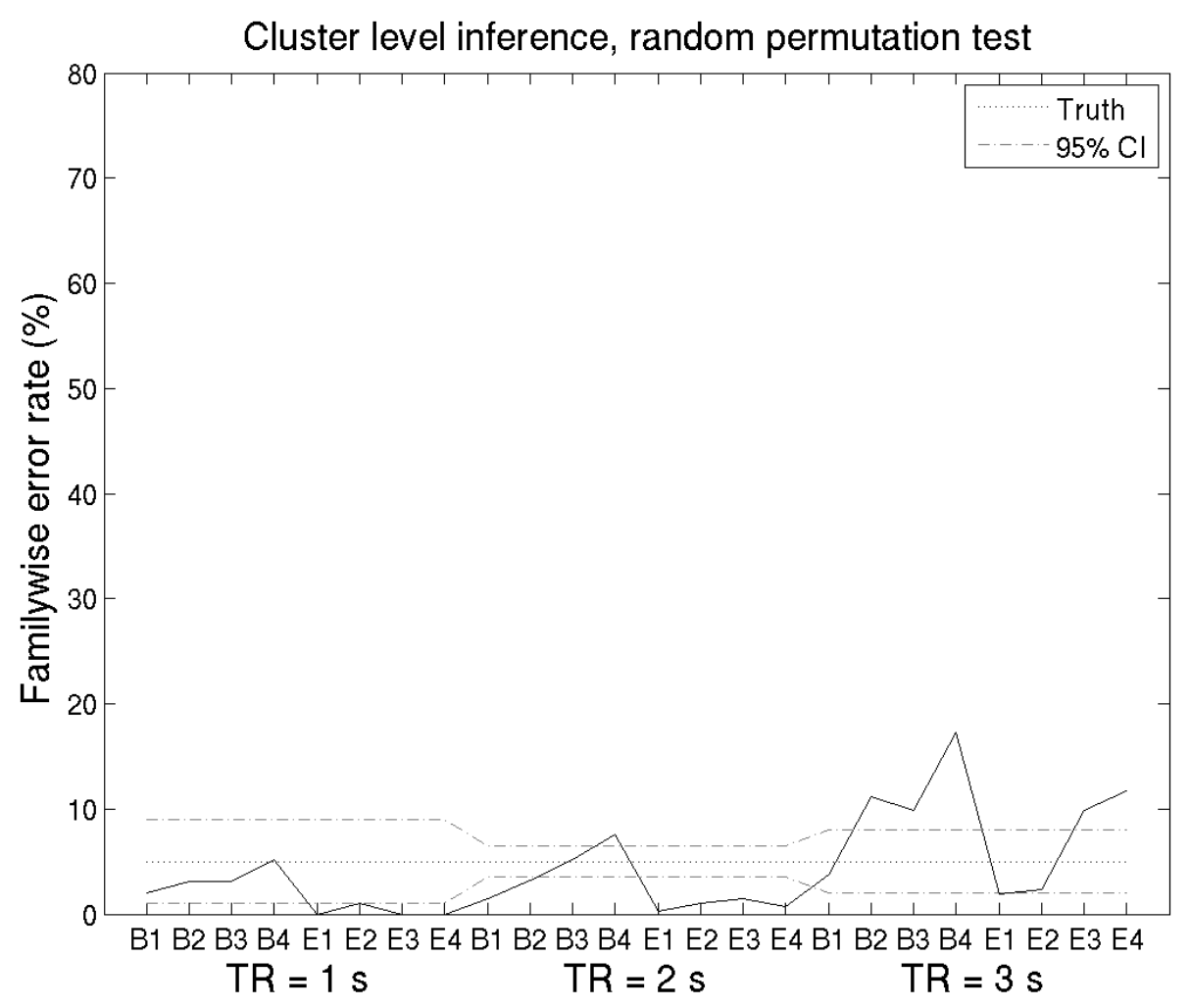

(b)

Figure 3: Familywise error rates for the random permutation test as function of paradigm and repetition time, for 8 mm of smoothing. The true familywise error rate of $5 \%$ and the $95 \%$ confidence intervals are included as reference. 


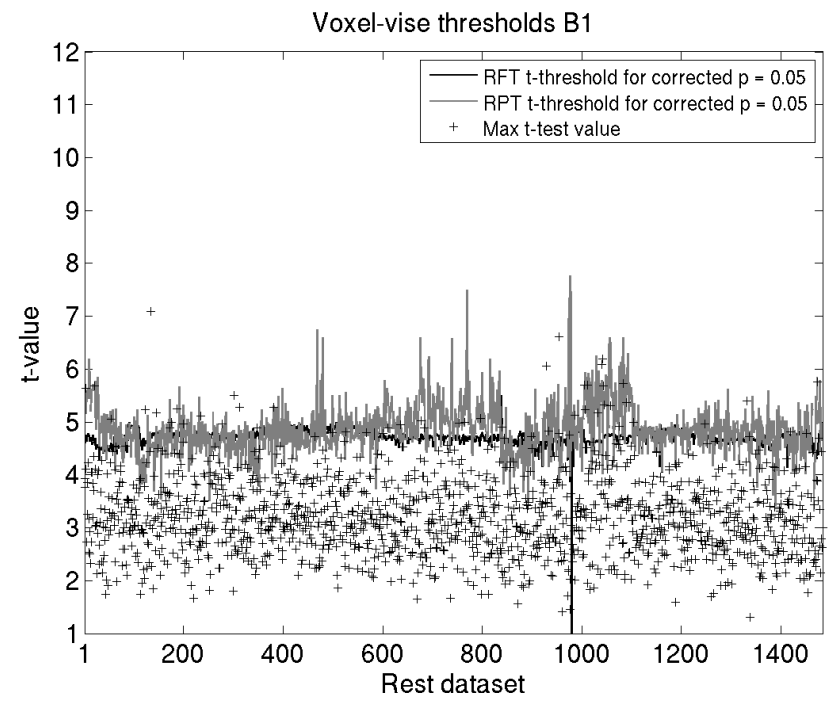

(a)

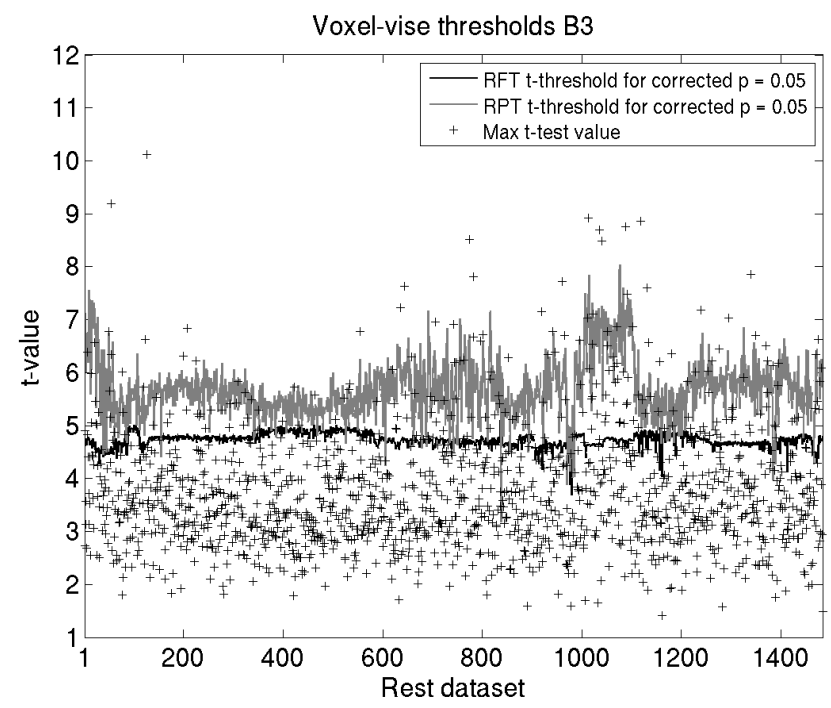

(c)

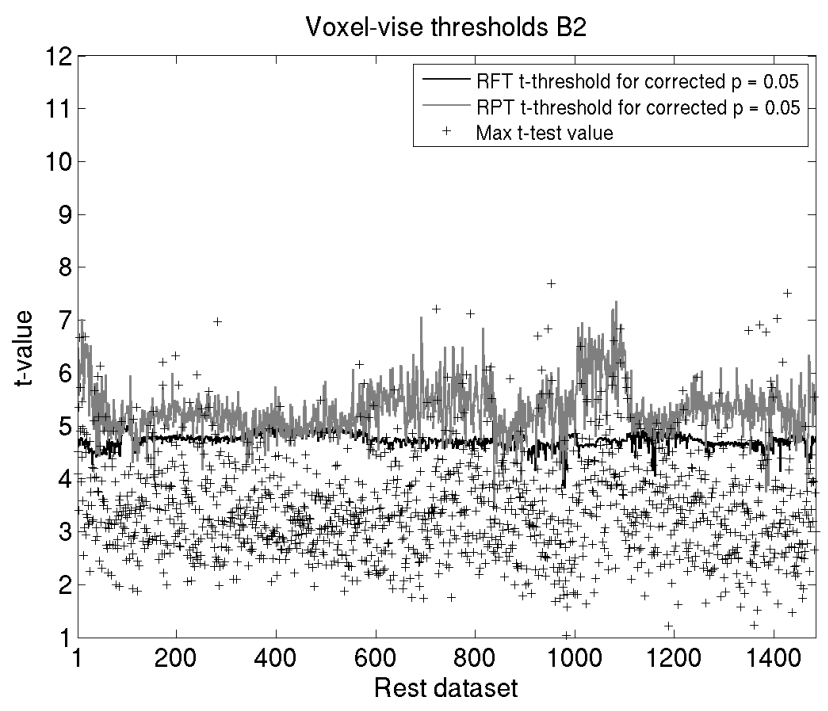

(b)

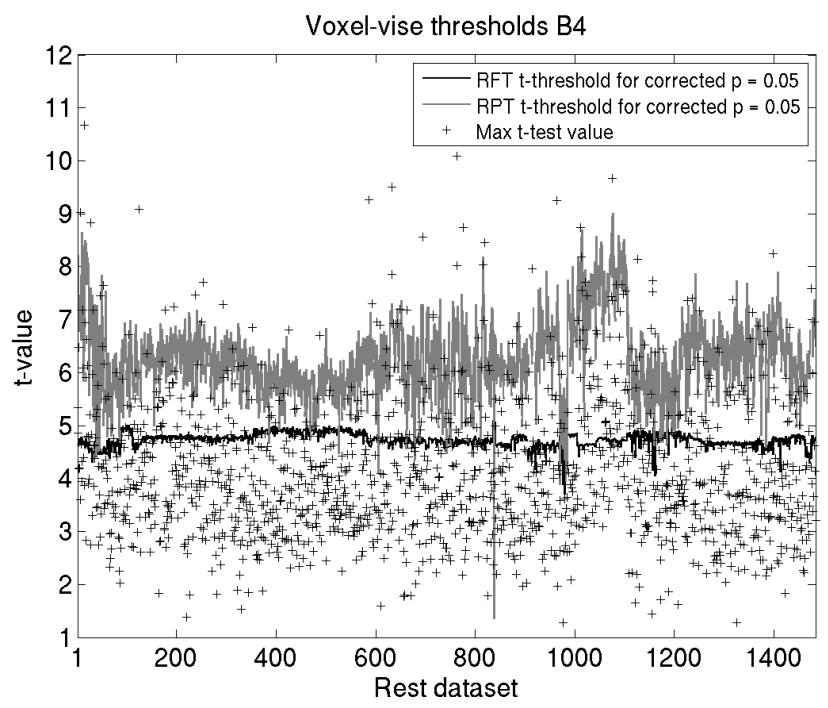

(d)

Figure 4: The maximum $t$-test values, the random field theory (RFT) $t$-thresholds and the random permutation test (RPT) $t$-thresholds, for block based designs and $8 \mathrm{~mm}$ of smoothing. Note that the significance thresholds from the random permutation test are highest for datasets with a repetition time of $1 \mathrm{~s}$ (datasets $1-59$ and $1005-1042)$ 


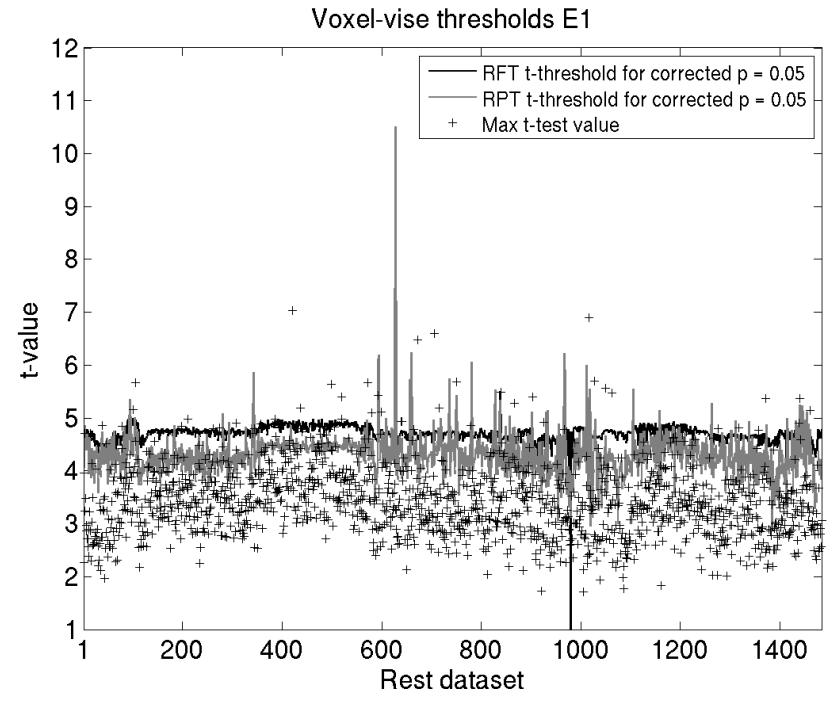

(a)

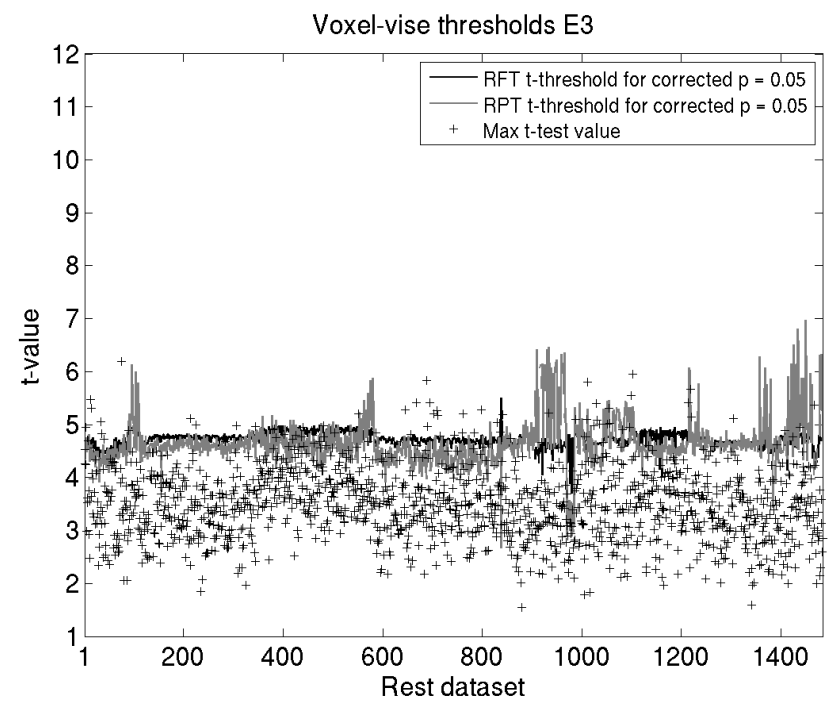

(c)

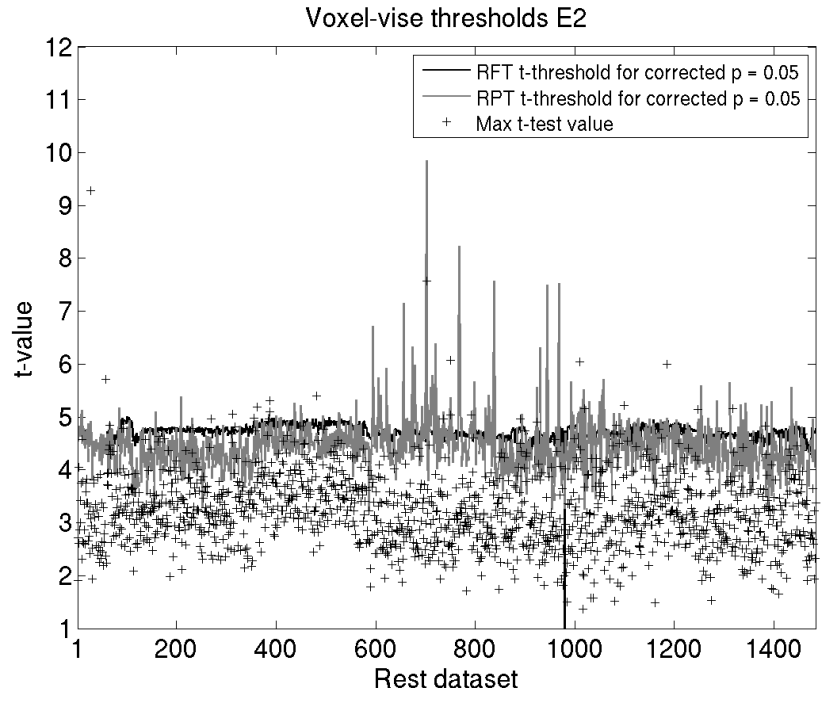

(b)

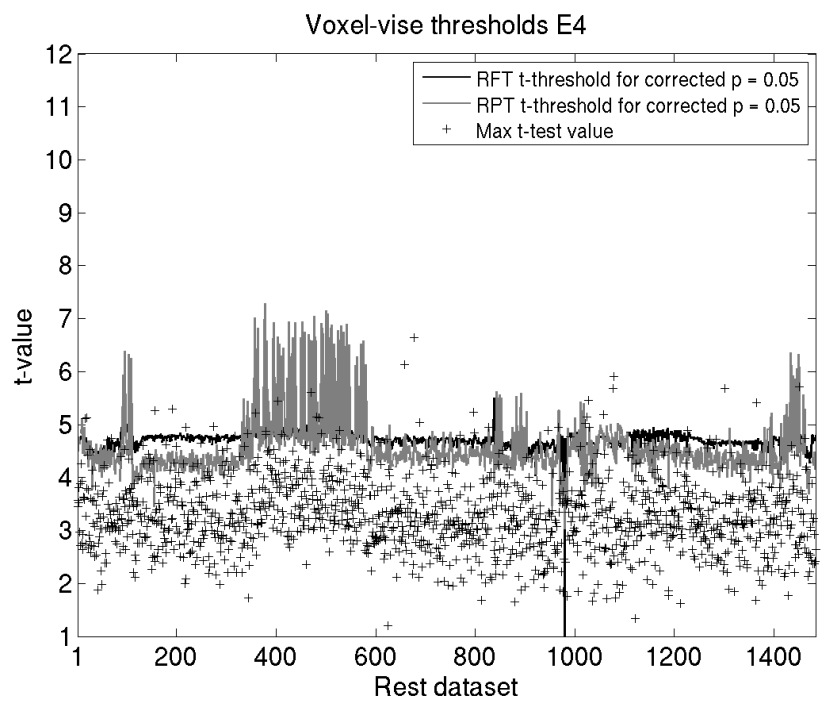

(d)

Figure 5: The maximum t-test values, the random field theory (RFT) t-thresholds and the random permutation test (RPT) t-thresholds, for event related designs and $8 \mathrm{~mm}$ of smoothing. 


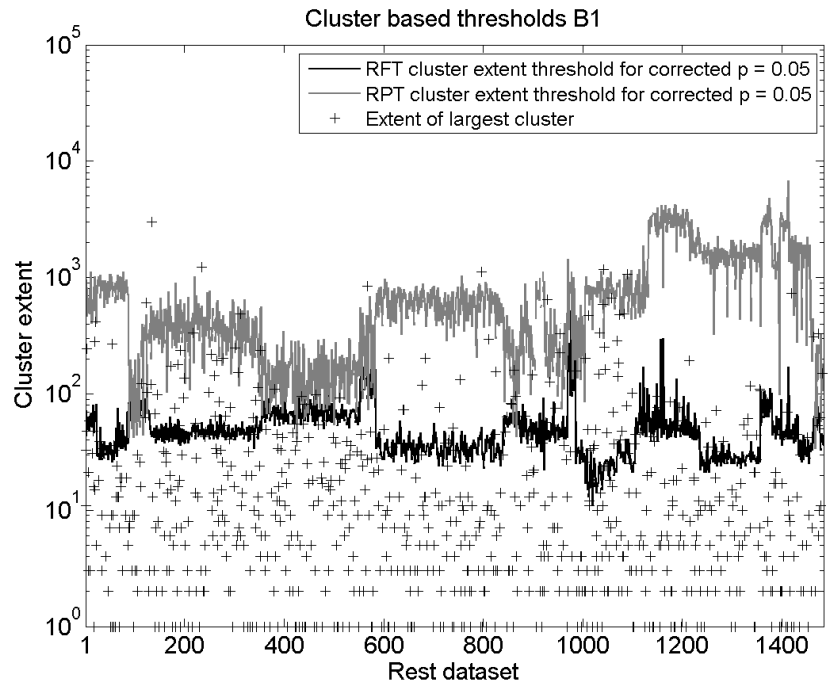

(a)

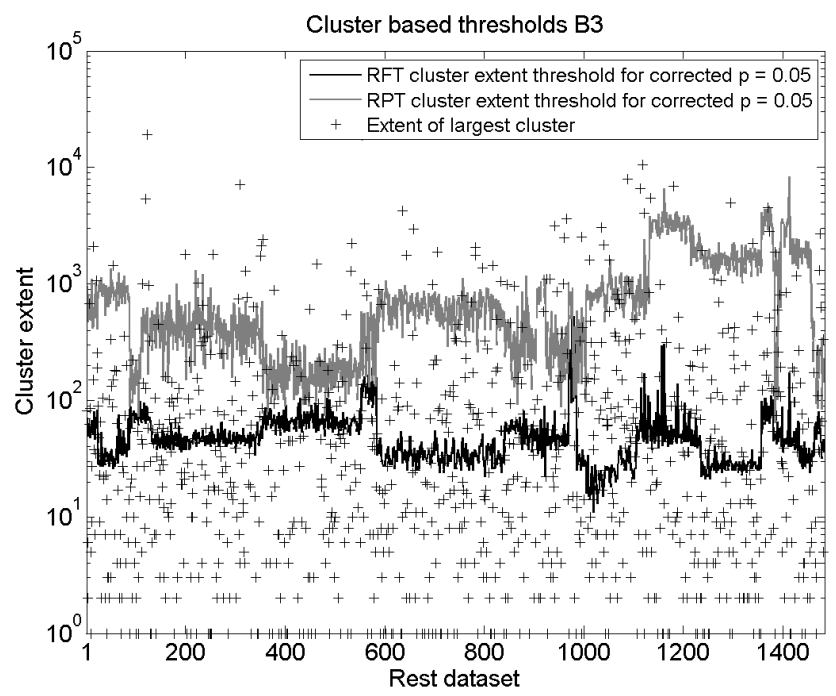

(c)

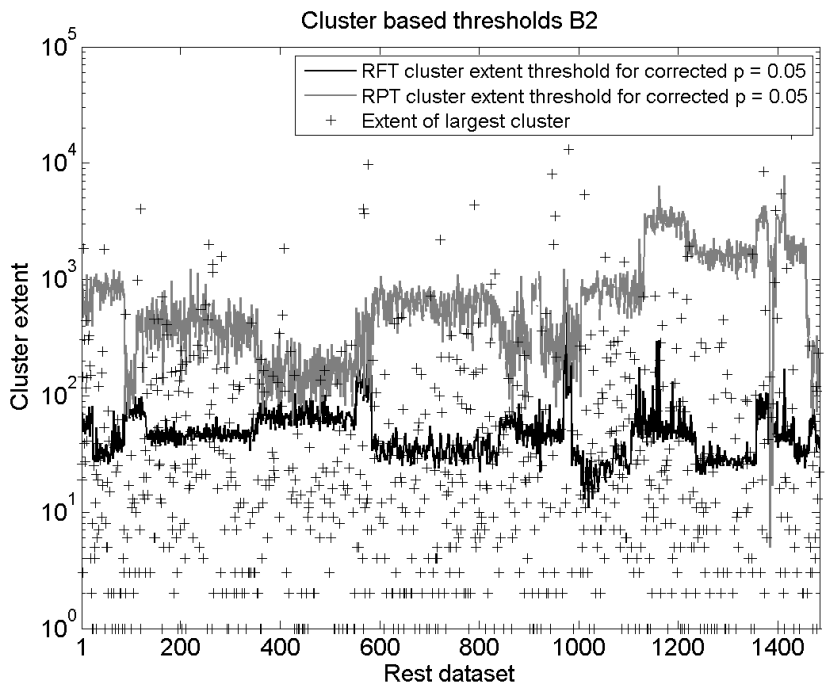

(b)

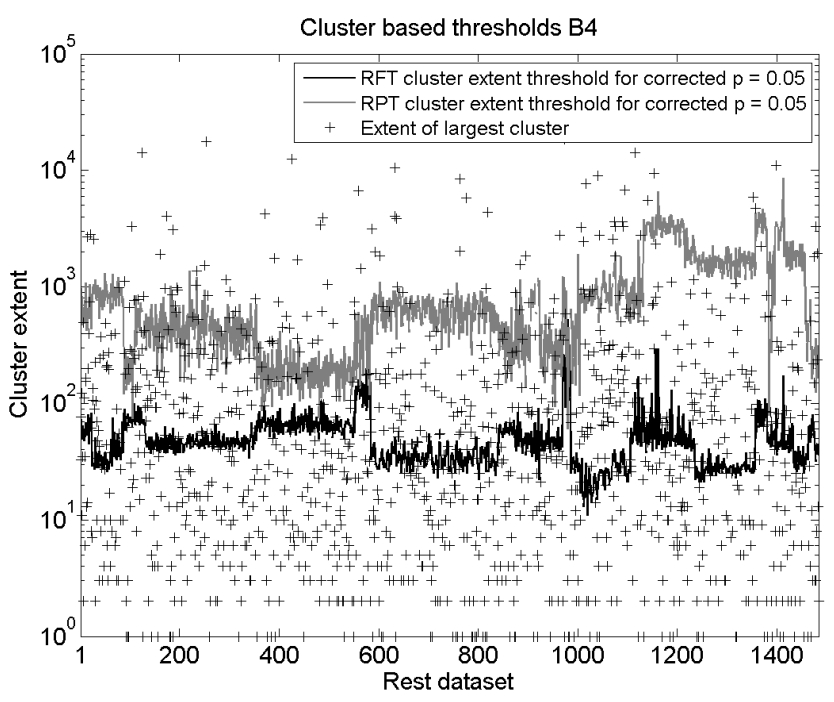

(d)

Figure 6: The extent of the largest clusters, the random field theory (RFT) cluster extent thresholds and the random permutation test (RPT) cluster extent thresholds, for block based designs and 8 mm of smoothing. 


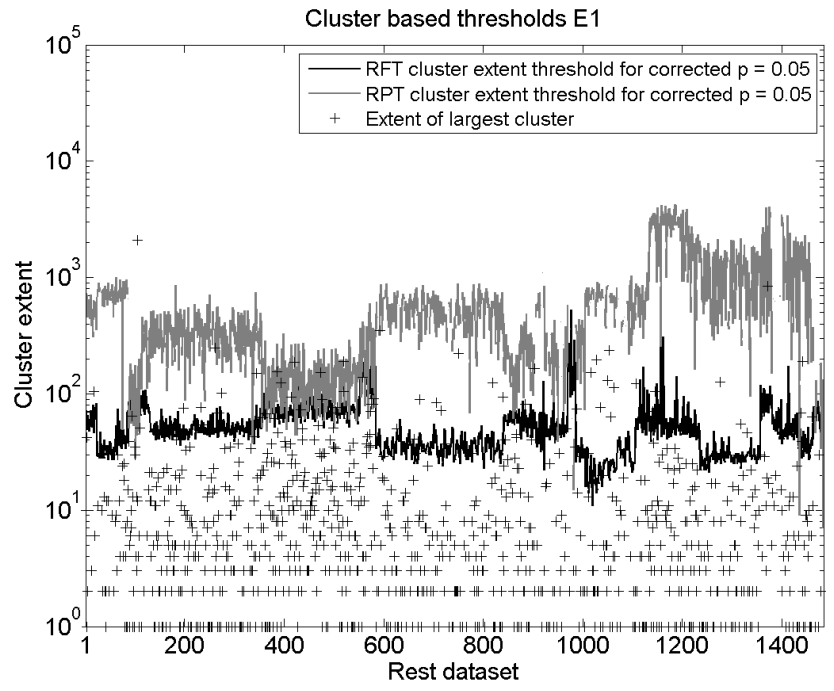

(a)

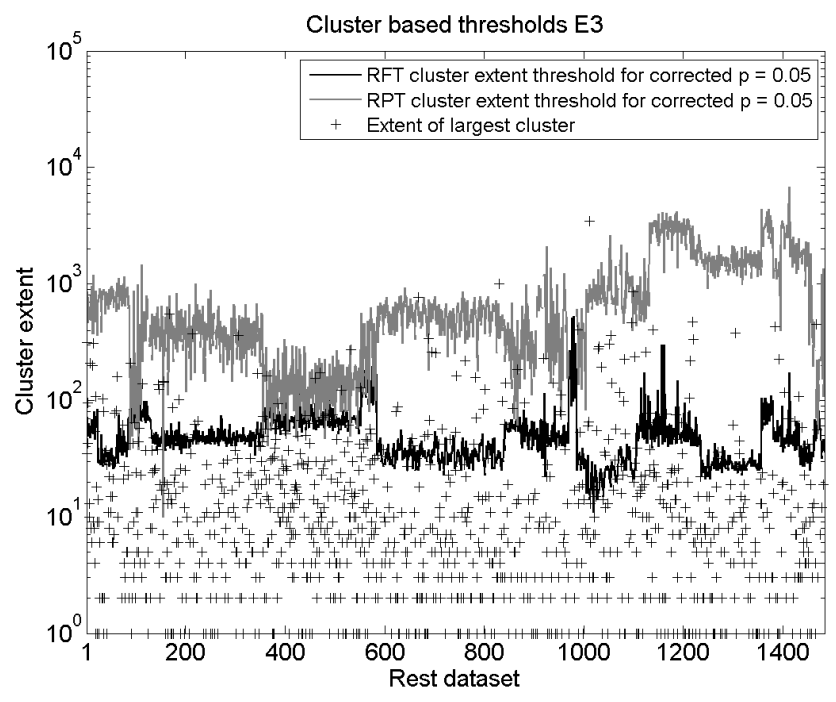

(c)

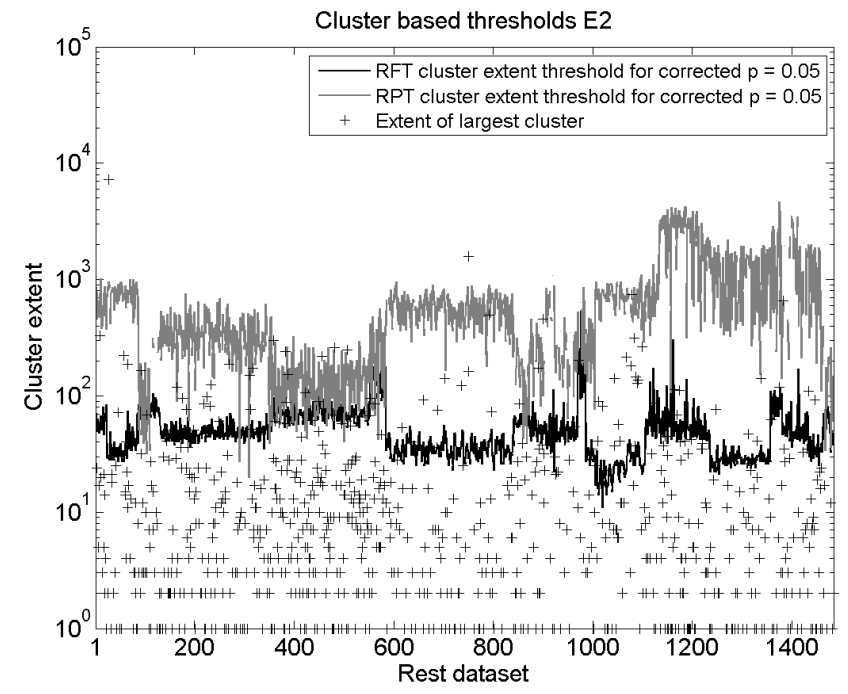

(b)

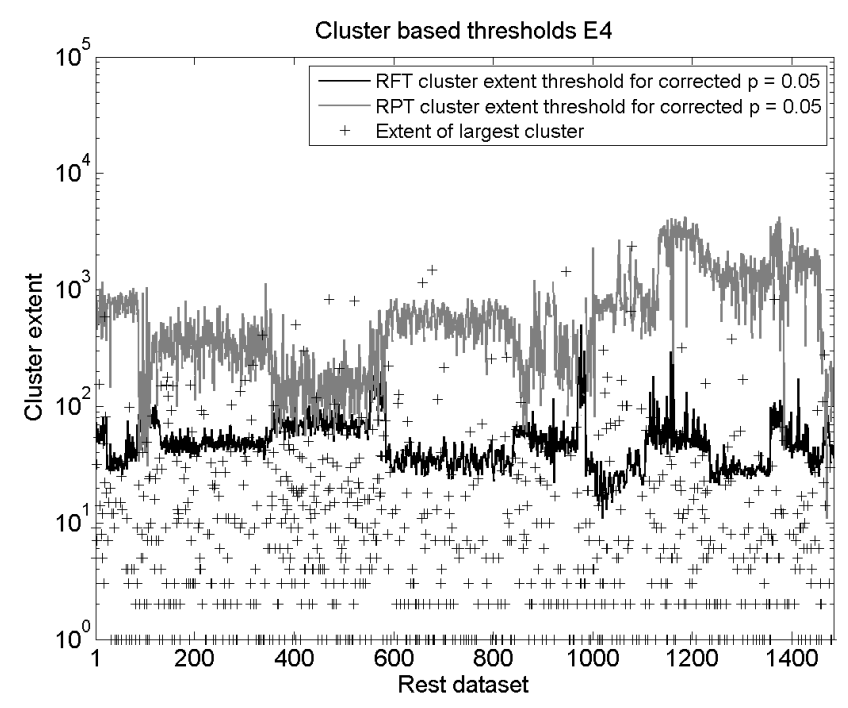

(d)

Figure 7: The extent of the largest clusters, the random field theory (RFT) cluster extent thresholds and the random permutation test (RPT) cluster extent thresholds, for event related designs and $8 \mathrm{~mm}$ of smoothing. 
Table 6: Results of the binary logistic regression analysis for SPM8, only significant parameter combinations are included. Four-way, five-way and six-way interaction was also tested, but did not yield any significant results.

\begin{tabular}{|l|c|c|c|}
\hline Parameter combination & Z-value & p-value & Odds ratio \\
\hline Smoothing & -6.86 & 0.000 & 0.56 \\
Paradigm & -14.08 & 0.000 & 0.20 \\
Repetition time & -21.50 & 0.000 & 0.11 \\
Inference level & 2.92 & 0.004 & 1.28 \\
Motion regressors & 2.59 & 0.010 & 1.25 \\
Two-way interactions & & & \\
Paradigm * Repetition time & & & \\
Paradigm * Motion regressors & 5.81 & 0.000 & 2.79 \\
Repetition time * Inference level & -3.21 & 0.001 & 0.58 \\
Three-way interactions & 6.51 & 0.000 & 2.34 \\
& & & \\
Paradigm * Repetition time * Inference level & & & \\
Paradigm * Repetition time * Motion regressors & -2.82 & 0.005 & 0.53 \\
Paradigm * Repetition time * Global normalization & -2.25 & 0.042 & 1.65 \\
\hline
\end{tabular}

Table 7: Results of the binary logistic regression analysis for the random permutation test.

\begin{tabular}{|l|c|c|c|}
\hline Parameter combination & Z-value & p-value & Odds ratio \\
\hline Paradigm & -2.40 & 0.016 & 0.43 \\
Repetition time & -2.65 & 0.008 & 0.49 \\
Inference level & -2.23 & 0.026 & 0.46 \\
Two-way interactions & & & \\
Paradigm * Repetition time & 4.87 & 0.000 & 7.58 \\
Paradigm * Inference level & -1.59 & 0.112 & 0.17 \\
Repetition time * Inference level & 4.76 & 0.000 & 6.98 \\
Three-way interaction & & & \\
Paradigm * Repetition time * Inference level & 0.03 & 0.976 & 1.03 \\
\hline
\end{tabular}


Average original spectrum for a repetition time of 1 second SPM8, no global normalization, no motion regressors

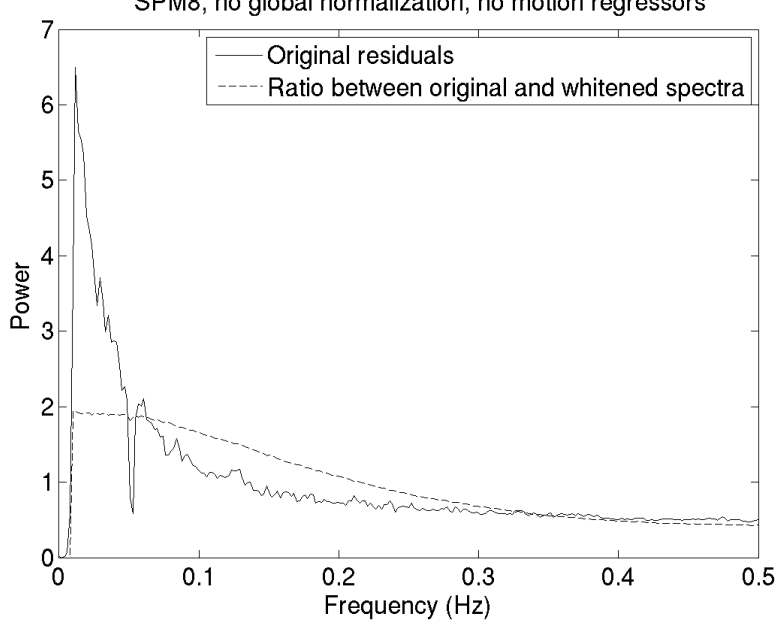

(a)

Average original spectrum for a repetition time of 2 seconds SPM8, no global normalization, no motion regressors

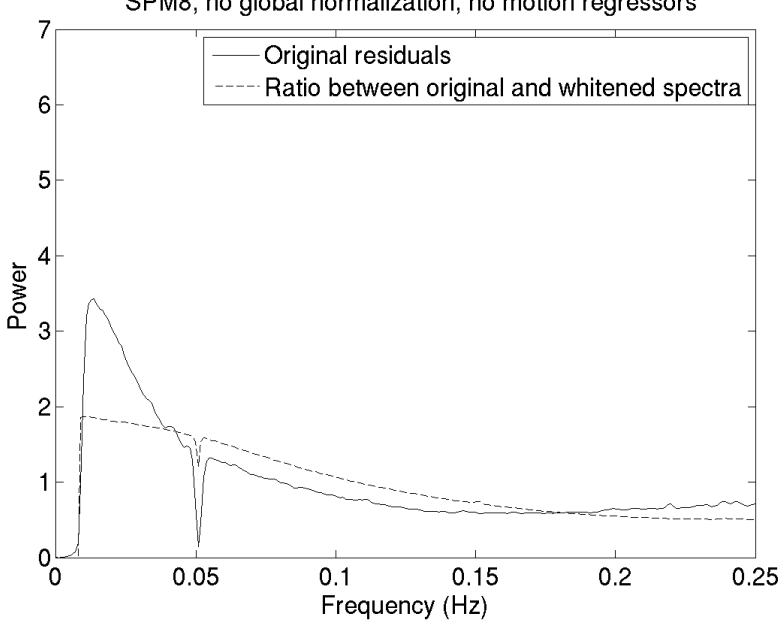

(b)

Average original spectrum for a repetition time of 3 seconds SPM8, no global normalization, no motion regressors

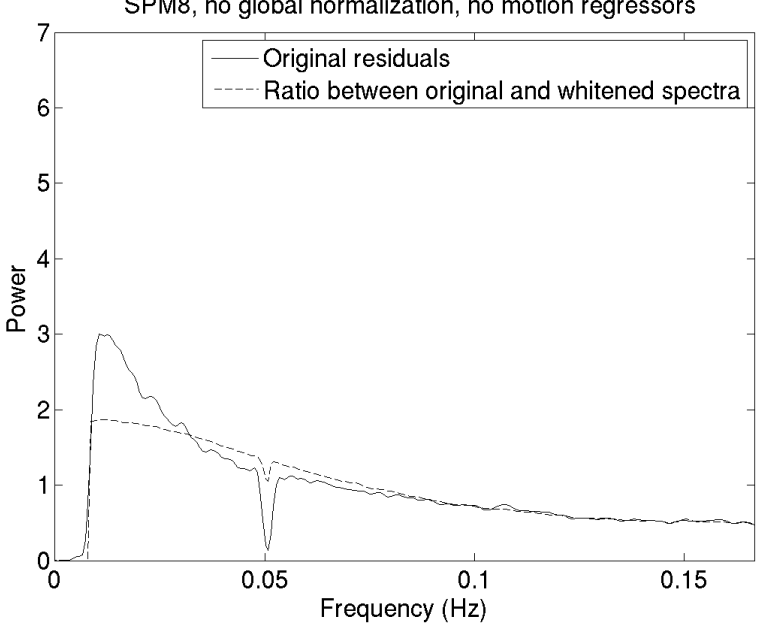

(c)

Figure 8: Average power spectra of standardized original residuals from SPM8 and ratio between the original and the whitened spectra, for different repetition times. Global normalization was not used and motion regressors were not included.
Average whitened spectrum for a repetition time of 1 second SPM8, no global normalization, no motion regressors

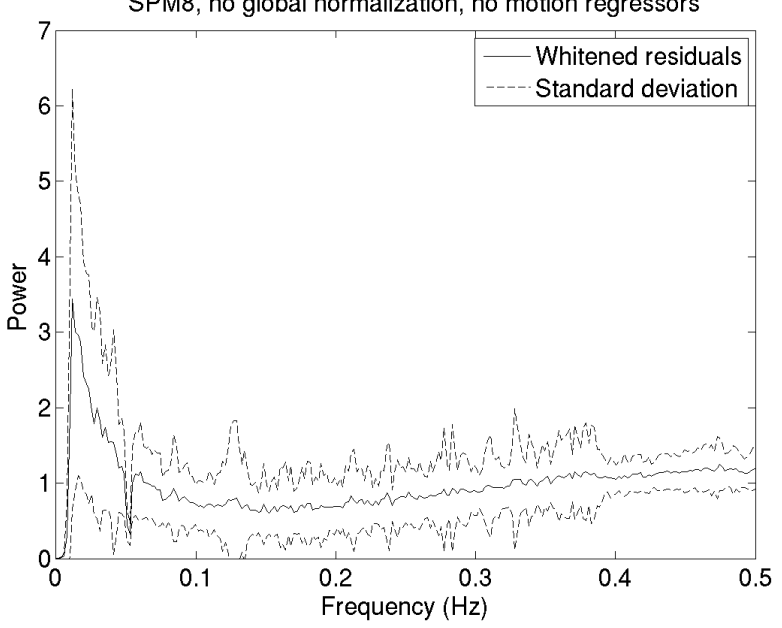

(a)

Average whitened spectrum for a repetition time of 2 seconds SPM8, no global normalization, no motion regressors

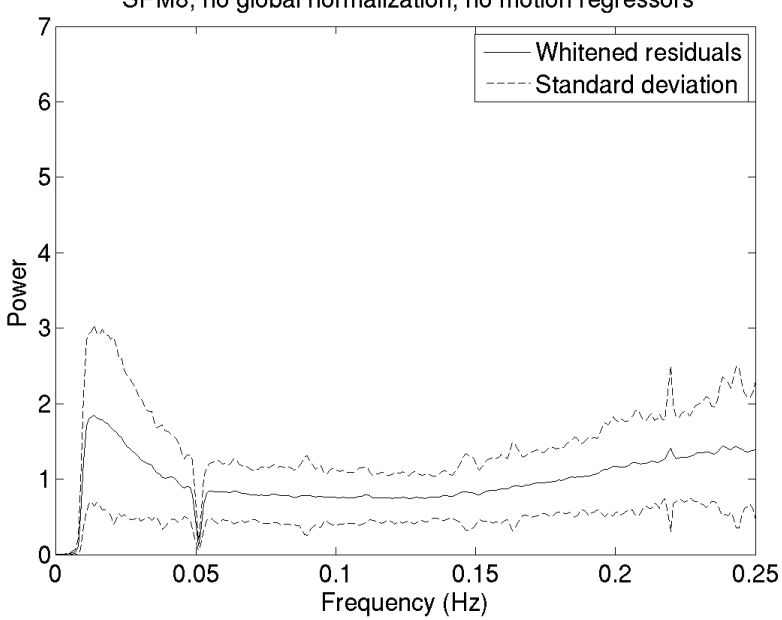

(b)

Average whitened spectrum for a repetition time of 3 seconds SPM8, no global normalization, no motion regressors

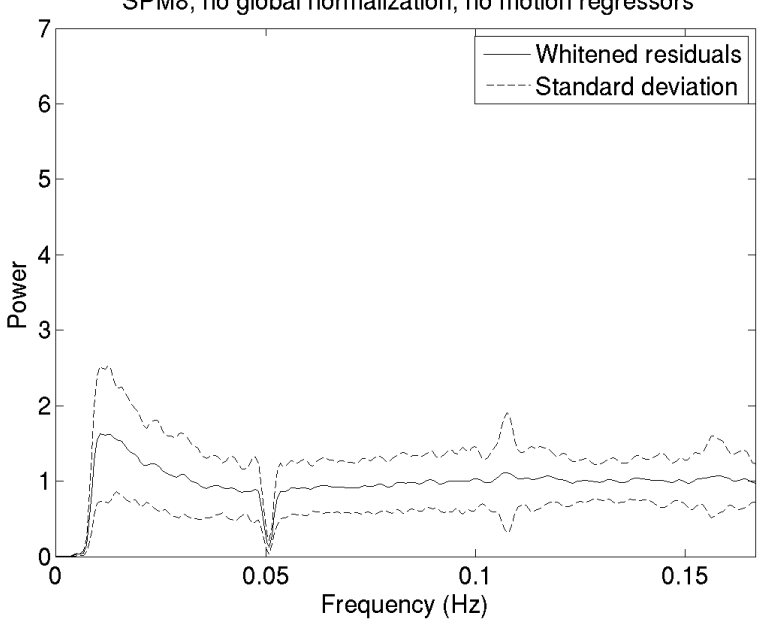

(c)

Figure 9: Average power spectra of standardized whitened residuals from SPM8, for different repetition times. Global normalization was not used and motion regressors were not included. 


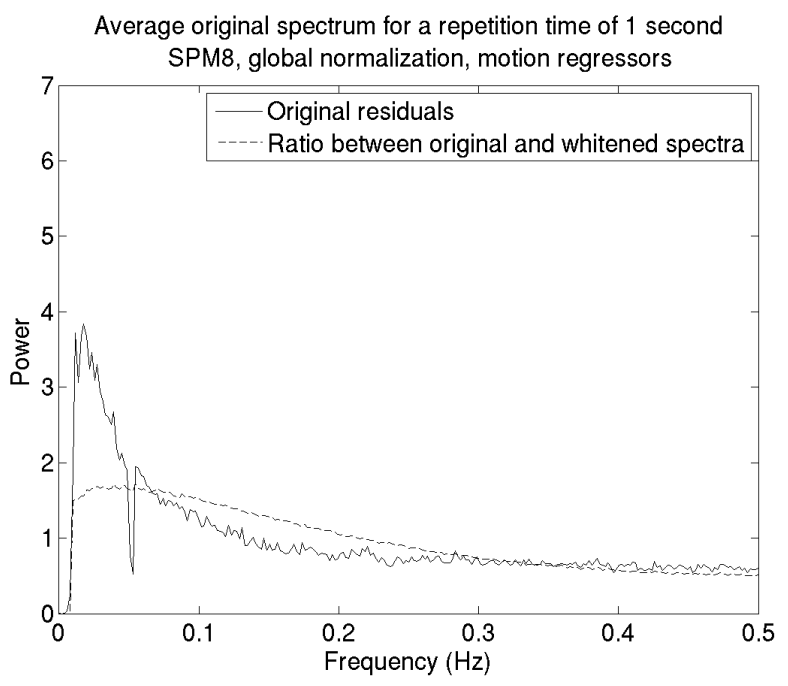

(a)

Average original spectrum for a repetition time of 2 seconds SPM8, global normalization, motion regressors

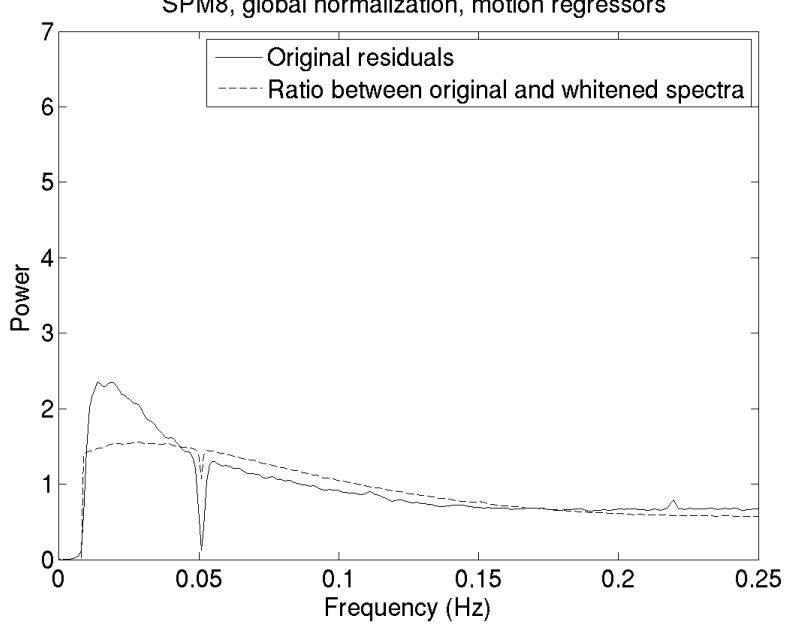

(b)

Average original spectrum for a repetition time of 3 seconds SPM8, global normalization, motion regressors

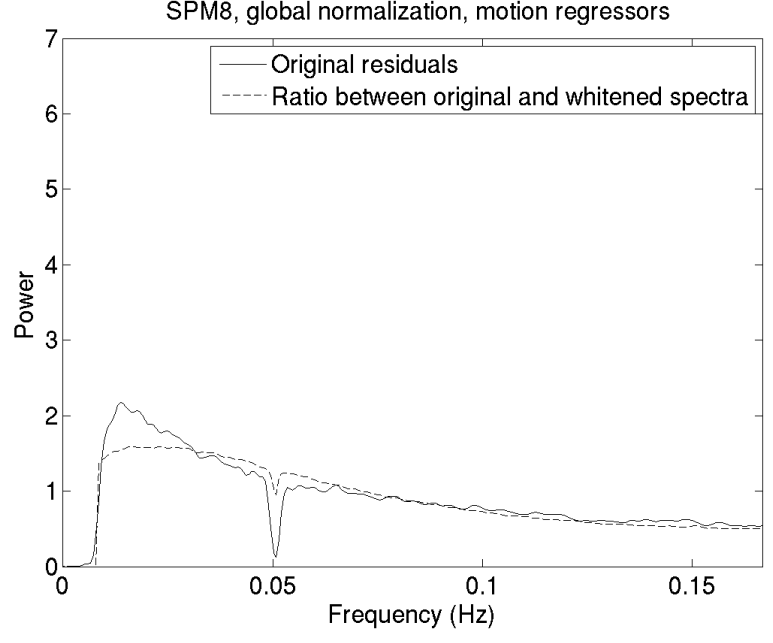

(c)

Figure 10: Average power spectra of standardized original residuals from SPM8 and ratio between the original and the whitened spectra, for different repetition times. Global normalization was used and motion regressors were included.

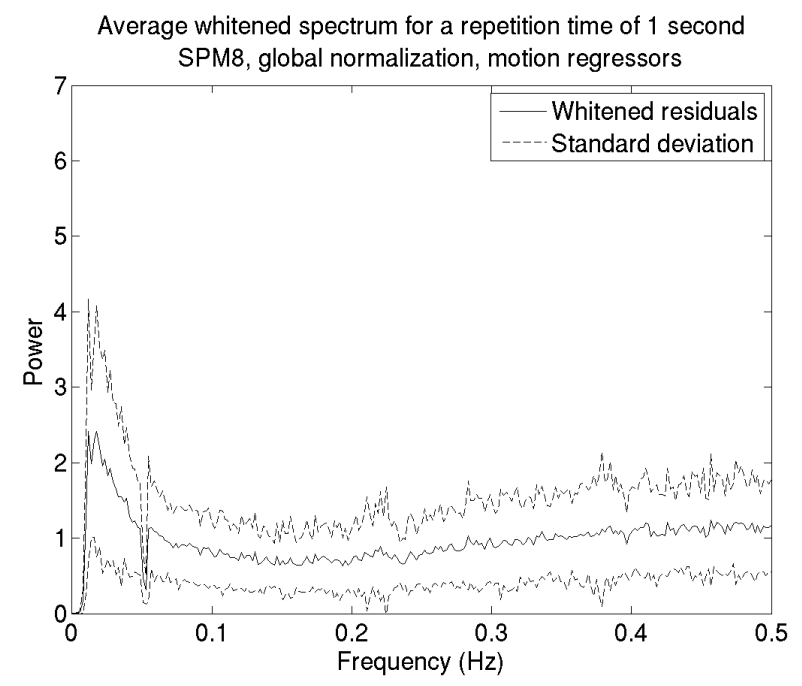

(a)

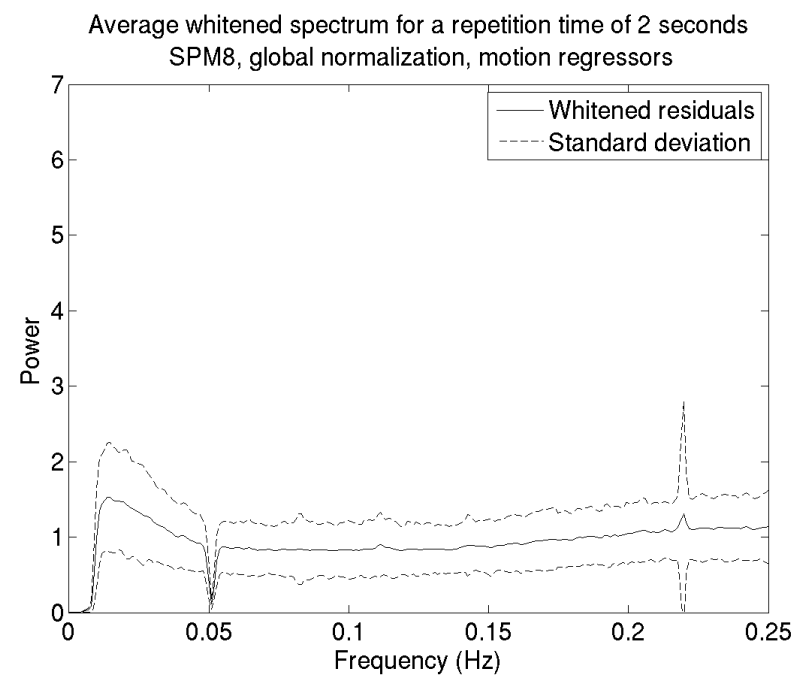

(b)

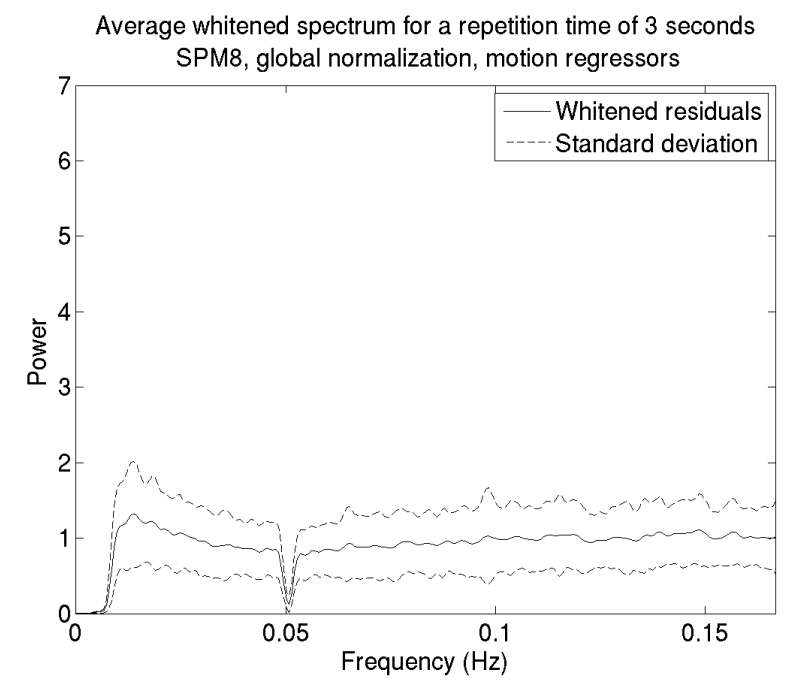

(c)

Figure 11: Average power spectra of standardized whitened residuals from SPM8, for different repetition times. Global normalization was used and motion regressors were included. 
Average original spectrum for a repetition time of 1 second Voxel-wise AR(4) whitening prior to permutations

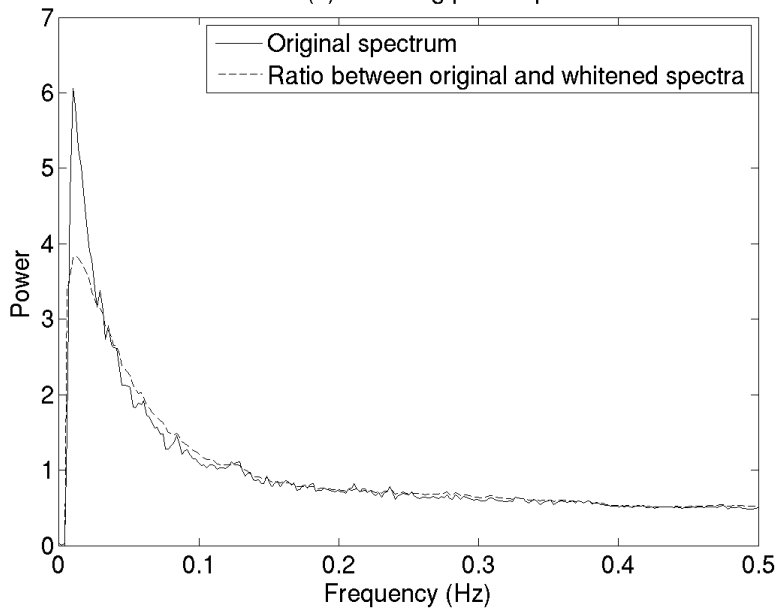

(a)

Average original spectrum for a repetition time of 2 seconds Voxel-wise $\mathrm{AR}(4)$ whitening prior to permutations

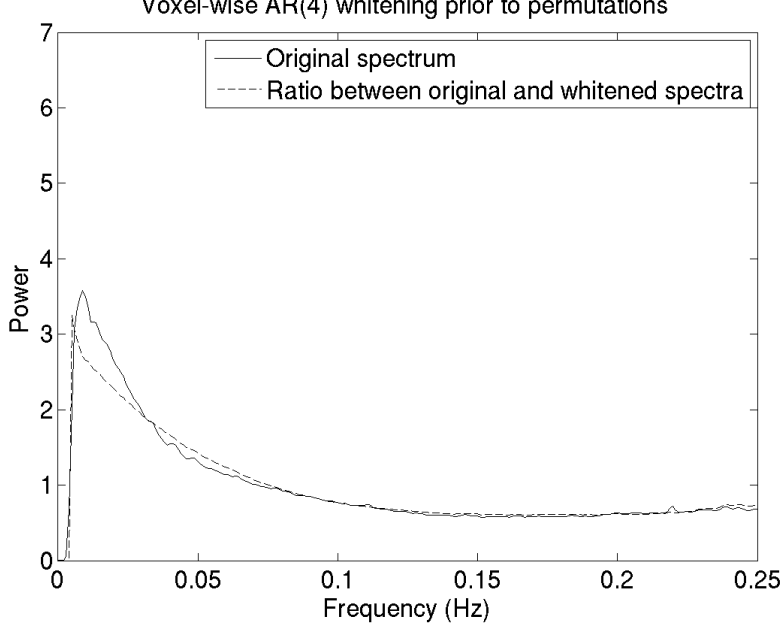

(b)

Average original spectrum for a repetition time of 3 seconds Voxel-wise $\operatorname{AR}(4)$ whitening prior to permutations

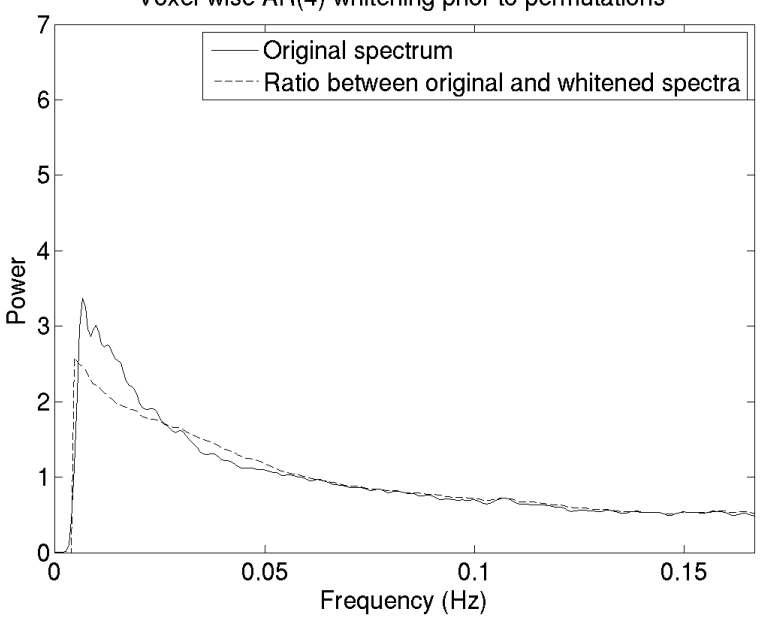

(c)

Figure 12: Average original power spectra and ratio between the original and whitened spectra, for different repetition times.
Average whitened spectrum for a repetition time of 1 second Voxel-wise AR(4) whitening prior to permutations

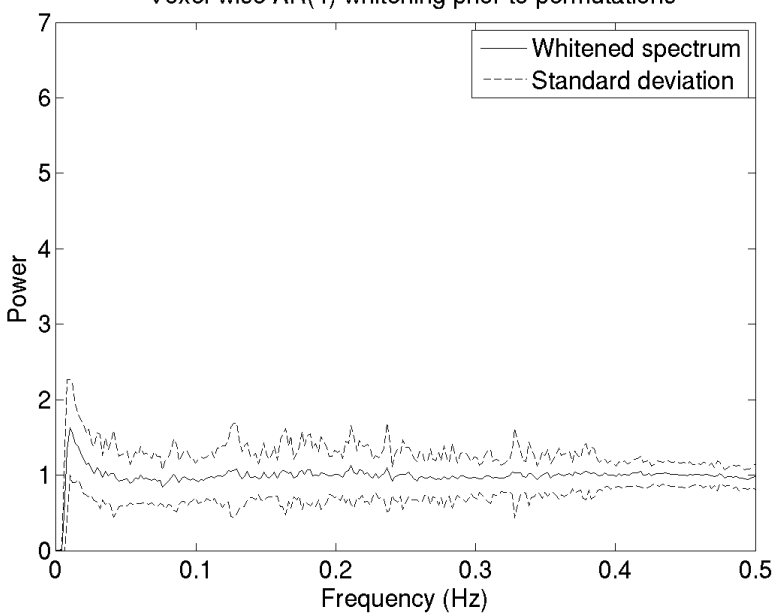

(a)

Average whitened spectrum for a repetition time of 2 seconds Voxel-wise AR(4) whitening prior to permutations

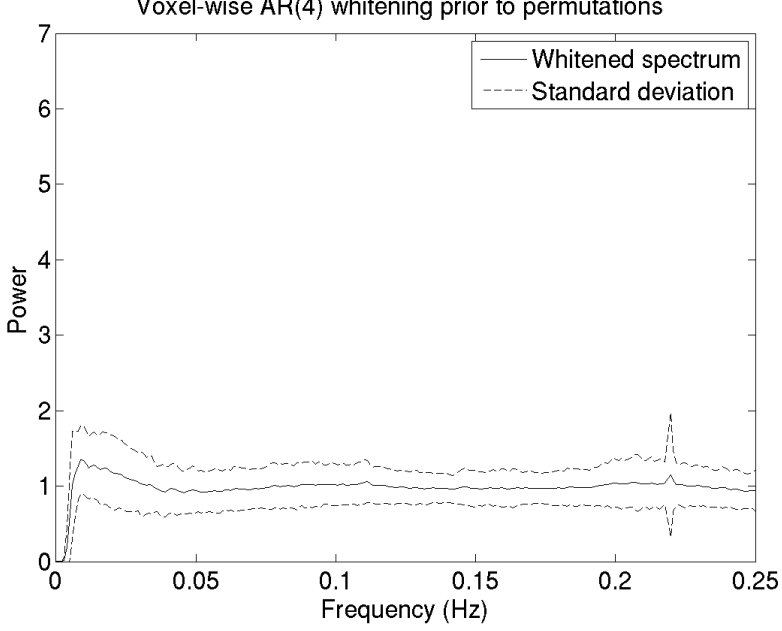

(b)

Average whitened spectrum for a repetition time of 3 seconds Voxel-wise $\operatorname{AR}(4)$ whitening prior to permutations

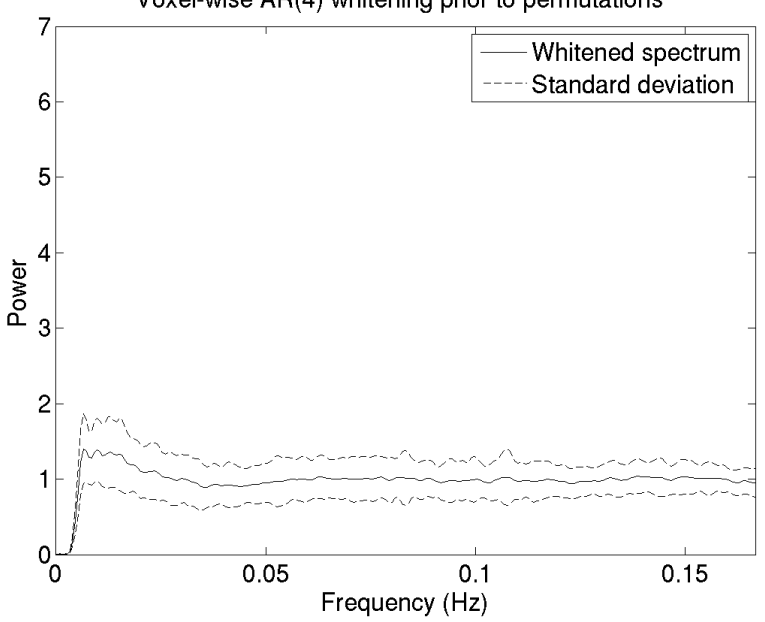

(c)

Figure 13: Average power spectra after whitening with a voxel-vise AR(4) model, for different repetition times. 


\section{Discussion}

In brief, our analysis of false positive rates reveals some striking and intuitive effects. Overall, a simple AR(1) model for temporal correlations appears to be adequate for fast designs (E1 and E2) at all three TRs. However, there is a massive inflation of false positive rates at short TRs that is particularly pronounced for slower (block) designs. At a TR of $1 \mathrm{~s}$, the false positive rate can reach up to $70 \%$ for block designs. The effect of smoothing is consistent and universal - increasing the smoothing reduces number of false positives. Furthermore, this effect is more pronounced at a shorter TR.

The results are intuitive if we look at the modelling of temporal correlations in the frequency domain. The spectra show a failure of the AR(1) model used in SPM to accommodate low frequencies, a failure that is exacerbated by short TRs. In other words, the AR(1) model fails to account for slow fluctuations in the residuals that appear to be more prevalent at short TRs. Our results are intuitive, because regressors (designs) with lower frequency components are clearly more sensitive to the failure of non-sphericity modelling in the low frequency range. In what follows we unpack these results and discuss their implications for future modelling work.

\subsection{Related studies}

We have only found one previous study that estimates familywise error rates using real data (Zarahn et al., 1997). The rest datasets were analyzed with a block based design with blocks of 40 seconds and the activity maps were thresholded at $\mathrm{p}=0.05$ (Bonferroni corrected for multiple testing). When independence was assumed between the time samples, activity was found in 10 out of 17 subjects. For a $1 / f$ auto correlation model, activity was found in 5 subjects. When the sequences were smoothed with an estimated BOLD impulse response function, and the $1 / f$ model of intrinsic auto correlation was included, activity was found in 1 subject. The same test without the auto correlation model resulted in activity in 3 subjects.

Another study on rest data from 8 subjects (Smith et al., 2007) used a block based design with blocks of 20 seconds and estimated voxel-wise error rates. When not performing whitening, an uncorrected threshold of $\mathrm{p}=0.001$ resulted in 862 false positives, compared to the expected 58 for 58000 brain voxels. When a global AR(1) whitening was applied (as in SPM8), the number of false positives dropped to 109. Similar results were found in another study (Purdon and Weisskoff, 1998) and the problem was found to be more severe for low frequency block based designs and short repetition times. A final example is a semi-parametric approach to calculate significance thresholds (Nandy and Cordes, 2007) which includes a discussion about the problems with low frequencies in resting state fMRI data. When rest data were analyzed with a gamma-convolved boxcar function with blocks of $30 \mathrm{~s}$ (B4), activity was found even after correcting for multiple testing. The random field theory $\mathrm{t}$-threshold was 4.70 and the semi-parametric approach resulted in a t-threshold of 6.61 .
The results of these studies are consistent with the results of the present study, but it is hard to draw strong conclusions as only a few datasets were used.

\subsection{Which parameters affect the familywise error rate?}

As can be seen in the plots and in the logistic regression analysis, the familywise error rate for SPM8 is significantly affected by the amount of smoothing $(p<0.0001, z=-6.86)$, the paradigm used $(\mathrm{p}<0.0001, \mathrm{z}=-14.08)$, the repetition time $(\mathrm{p}<0.0001, \mathrm{z}=-21.50)$, the inference level $(\mathrm{p}=0.004)$ and if motion regressors are used or not $(\mathrm{p}=0.01)$. There is also two-way interactions between paradigm and repetition time $(\mathrm{p}$ $<0.0001, \mathrm{z}=5.81$ ), between paradigm and motion regressors $(\mathrm{p}=0.001)$ and between repetition time and inference level $(\mathrm{p}<0.0001, \mathrm{z}=6.51)$. Three-way interaction was found between paradigm, repetition time and inference level $(\mathrm{p}=0.005)$, between paradigm, repetition time and motion regressors $(\mathrm{p}=$ 0.042 ) and between paradigm, repetition time and global normalization ( $\mathrm{p}=0.024)$. If multiple testing is considered, and each test is seen as independent (i.e. Bonferroni adjustment), smoothing, paradigm and repetition time are still significant. The two-way interactions between paradigm and repetition time and between repetition time and inference level are also still significant.

The random permutation test is also significantly affected by the repetition time $(\mathrm{p}=0.008)$, the paradigm $(\mathrm{p}=0.016)$ and the inference level $(p=0.026)$. The $z$-values for these parameters are, however, lower than for SPM8.

\subsection{Non-white noise}

The familywise error rates are higher for block based designs with longer periods; this is consistent with the $1 / f$ model that is often used for fMRI noise (Zarahn et al., 1997; Smith et al., 1999; Friston et al., 2000). One problem in fMRI is that the sampling rate normally is too low to accurately represent physiological noise, such as breathing and heartbeats (Mitra and Pesaran, 1999; Dagli et al., 1999; Lund et al., 2006). Temporal aliasing is thereby introduced, which invalidates the $1 / f$ model. Aliasing is probably the reason why the residuals have relatively high energy for high frequencies. The familywise error rates are higher for short repetition times, which previously has been reported for voxel-vise error rates (Purdon and Weisskoff, 1998). This is explained by the fact that the auto correlation of a signal, as function of the sample distance, increases with the sampling frequency (Purdon and Weisskoff, 1998) (but the auto correlation as function of the time distance is constant). As subsecond repetition times are becoming possible in fMRI (Feinberg et al., 2010), it is rather alarming that results from SPM are less valid for short repetition times.

The non-white noise (Friman et al., 2005; Lund et al., 2006) can yield p-values that are too low (Purdon and Weisskoff, 1998; Lund et al., 2006; Smith et al., 2007). SPM uses high pass filtering as a first remedy. One could remove more of the low frequencies, by increasing the cutoff frequency of the high pass filter. This can, however, increase the number of false positives even further (Smith et al., 2007). After the high pass filtering, 
a global AR(1) auto correlation correction is applied (Friston et al., 2000). The reason why the same AR parameter is used for all the brain voxels, is that the effective degrees of freedom varies between the voxels if an individual whitening is used. As can be seen in Figures 9(a) - 9(c) and 11(a) - 11(c), the global AR(1) model used in SPM fails to whiten the residuals for short repetition times. An explanation for this can be that the SPM software was designed when it was common to use very long repetition times, for which the global AR(1) whitening works rather well. Other software packages for fMRI analysis (e.g. FSL, AFNI, fmristat) use more sophisticated modelling of the auto correlation and may potentially yield familywise error rates that are closer to the expected ones.

Our work suggests a need to improve, or extend, the models of temporal correlations or stationary dependencies in single subject fMRI timeseries. This is a non-trivial problem, since one cannot simply estimate the auto correlation function of the residuals. This follows from the fact that one needs to estimate the non-sphericity of the underlying random errors, as opposed to the residuals of a general linear model. However, one cannot simply measure the auto correlations in the raw data, because these include dependencies due to signal. This is why one has to use estimates (for example restricted maximum likelihood estimators) of the underlying smoothness by making particular assumptions about the form of the unobserved correlations among the real errors. Here, the assumption is temporal stationarity, which allows us to represent the non-sphericity in terms of an auto correlation function or spectral density. The problem of non-sphericity is made more acute by the fact that estimating auto correlation functions, from single voxel timeseries, can lead to inefficient (variable) estimates. This is why we smoothed the estimated $\mathrm{AR}(4)$ coefficients in the non-parametric analyses, as for example proposed by (Worsley et al., 2002). In summary, the advent of very short TR capabilities (Feinberg et al., 2010) may call for a re-appraisal of existing assumptions about the form and stationarity of temporal correlations in fMRI.

\subsection{Non-parametric fMRI analysis}

If the exact noise structure was known, a lot of problems in fMRI would be solved, but not all of them. Non-parametric fMRI analysis (Siegel, 1957; Dwass, 1957; Holmes et al., 1996; Brammer et al., 1997; Bullmore et al., 2001; Nichols and Holmes, 2001; Nichols and Hayasaka, 2003; Tillikainen et al., 2006; Eklund et al., 2011a) can be required in order to calculate significance thresholds and p-values for detection statistics that are more advanced than the GLM, for example multi-voxel approaches, which do not necessarily have a known parametric null distribution (Friman et al., 2001, 2003; Nandy and Cordes, 2003; Mourao-Miranda et al., 2005; Kriegeskorte et al., 2006; Norman et al., 2006; Martino et al., 2008; Björnsdotter et al., 2011). The beauty of the random permutation test is that it can be used to calculate significance thresholds and p-values for any test statistics, for example fMRI analysis by restricted canonical correlation analysis (Das and Sen, 1994; Friman et al., 2003; Eklund et al., 2011a, 2012).
As previously mentioned, the thresholds from the random permutation test cannot directly be compared to the RFT thresholds, as the preprocessing and the statistical analysis is not performed exactly as in SPM8. It is, however, clear that the random permutation test, for voxel level inference, gives higher thresholds for the block based designs and slightly lower thresholds for the event related designs. Note that the significance thresholds from the random permutation test, are highest for datasets with a repetition time of $1 \mathrm{~s}$ (datasets 1 - 59 and 1005 - 1042). The reason why the random permutation test works better for block based designs, than for event related designs, is probably that the regressors for the randomized event related designs (E3 and E4) have a wider spectra than the other regressors. These designs are thereby more sensitive to that the whitening prior to the permutations is correct.

For cluster level inference, the thresholds from the random permutation test are in general too high. A possible explanation for this is that cluster based thresholds are more sensitive to a perfect whitening, than voxel-wise thresholds. The whitened spectra, Figures 13(a) - 13(c), are rather flat, but this does not necessarily mean that the whitening works for all datasets (and timeseries). It only means that the whitening works well on average. The standard deviation of the whitened spectra from the voxel-vise AR(4) whitening is clearly smaller than for the whitened spectra from SPM.

\subsection{Rest vs activity data}

It is not straight forward to generalize our findings to standard analyses of activation studies with SPM8. This is because resting state data was analyzed, which deliberately promotes slow fluctuations in activity (to estimate functional connectivity or coherence at low temporal frequencies). This means that the residuals may be dominated by low frequencies that confound standard (simple AR) models of serial correlations. A solution to this problem could be to analyse activity data with a regressor that is orthogonal to the used paradigm. To give an example, if fMRI activity data has been collected with a block based design, analyse the data with an event related design and count the number of "false positives". Data from the OpenfMRI project,

$$
\text { http://www.openfmri.org, }
$$

can be used for this purpose.

\subsection{Computational complexity}

To analyze an fMRI dataset with 7 amounts of smoothing and 8 statistical designs on average takes 10 minutes with SPM8, on an Intel Core i7 3,4 GHz with 16 GB of memory. For 1482 datasets this gives a total of 82992 analyses and a processing time of about 10 days. The analysis was done with and without global normalization and motion regressors, yielding a total of 331968 analyses. By instead using the computational power of the graphics processing unit (GPU) (Gembris et al., 2011; A. R. Ferreira da Silva, 2011, 2010; Eklund et al., 2011a, 2012, $2011 \mathrm{~b}$ ) the processing time can be reduced to 5 - 10 seconds per dataset, giving a total processing time of 2 - 4 hours. 
The main drawback of non-parametric statistical approaches is their computational complexity, which so far has limited their use in fMRI. Thresholding techniques for single subject fMRI are more complicated than for multi subject fMRI, as the fMRI time series contain auto correlation (Woolrich et al., 2001). To be able to perform a permutation test on single subject fMRI data, the auto correlations have to be removed prior to the resampling (Locascio et al., 1997; Bullmore et al., 2001; Friman and Westin, 2005), in order to not violate the exchangeability criterion. Single subject fMRI is further complicated by the fact that the spatial smoothing changes the auto correlation structure of the data. This problem is more obvious for CCA based fMRI analysis, where several filters are applied to the fMRI volumes (Friman et al., 2003). The only solution to always have null data with the same properties, is to perform the spatial smoothing in each permutation, which significantly increases the processing time. This problem was recently solved, by doing random permutation tests on the GPU (Eklund et al., 2011a, 2012). A random permutation test with 10000 permutations, for the 8 statistical designs, takes 5-15 minutes per dataset with a multi-GPU implementation, giving a total processing time of about 10 days. Note that 10000 permutations of $85 \mathrm{~GB}$ of data is equivalent to analyse $850 \mathrm{~TB}$ of data. To perform 11856 permutation tests ( 1482 datasets $\times 8$ paradigms) with SPM8 would take something like 100 years. We believe that the GPU will become an important tool for fMRI analysis.

\subsection{Future work}

This study has only considered single subject fMRI analysis, but the problems of non-white noise can also affect the results of a second-level analysis (Bianciardi et al., 2004). We therefore intend to repeat the empirical study for multi-subject fMRI. It would also be interesting to repeat the study with other programs for fMRI analysis, such as FSL, AFNI and fmristat, to see if the more advanced auto correlation modelling results in more accurate familywise error rates.

To improve the random permutation test, it is possible to use noise models that are temporally non-stationary (Milosavljevic et al., 1995; Long et al., 2005; Luo and Puthusserypady, 2007). The important aspect is that there now exists an objective way to compare the correctness of different parametric and non-parametric approaches.

\section{Conclusions}

We have presented the results of an empirical study, based on 1484 rest datasets, which shows that parametric fMRI analysis with SPM can give invalid results. The results that are reported in this paper can, however, not be generalized to parametric fMRI analysis in general, other fMRI software packages may give different results. The random permutation test works well in some cases, but indicates that more advanced whitening is necessary. We challenge other researchers to get better results, and encourage them to repeat the study to verify our findings. To facilitate this, we have put all the datasets, the Matlab scripts and the results at
http://people.imt.liu.se/andek/rest_fMRI/ .

\section{Acknowledgement}

This work was supported by the Linnaeus center CADICS, funded by the Swedish research council, and by the Neuroeconomic research group at Linköping University. NovaMedTech is acknowledged for financial support of the GPU hardware.

The authors would like to thank the Neuroimaging Informatics Tools and Resources Clearinghouse (NITRC) and all the institutions that have contributed with data to the 1000 functional connectomes project. Without their efforts this empirical study would not have been possible.

\section{References}

A. R. Ferreira da Silva, 2010. cudaBayesreg: Bayesian Computation in CUDA. The R Journal 2/2, 48-55.

A. R. Ferreira da Silva, 2011. A Bayesian multilevel model for fMRI data analysis. Computer Methods and Programs in Biomedicine 102, 238-252.

Bennett, C.M., Baird, A.A., Miller, M.B., Wolford, G.L., 2010. Neural correlates of interspecies perspective taking in the post-mortem atlantic salmon: An argument for multiple comparisons correction. Journal of Serendipitous and Unexpected Results 1, 1-5.

Bianciardi, M., Cerasa, A., Patria, F., Hagberg, G., 2004. Evaluation of mixed effects in event-related fMRI studies: Impact of first-level design and filtering. NeuroImage 22, 1351-1370.

Biswal, B., Mennes, M., Zuo, X.N., Gohel, S., Kelly, C., Smith, S.M., Beckmann, C.F., Adelstein, J.S., Buckner, R.L., Colcombe, S., Dogonowski, A.M., Ernst, M., Fair, D., Hampson, M., Hoptman, M.J., Hyde, J.S., Kiviniemi, V.J., Kötter, R., Li, S.J., Lin, C.P., Lowe, M.J., Mackay, C., Madden, D.J., Madsen, K.H., Margulies, D.S., Mayberg, H.S., McMahon, K., Monk, C.S., Mostofsky, S.H., Nagel, B.J., Pekar, J.J., Peltier, S.J., Petersen, S.E., Riedl, V., Rombouts, S.A., Rypma, B., Schlaggar, B.L., Schmidt, S., Seidler, R.D., Siegle, G.J., Sorg, C., Teng, G.J., Veijola, J., Villringer, A., Walter, M., Wang, L., Weng, X.C., Whitfield-Gabrieli, S., Williamson, P., Windischberger, C., Zang, Y.F., Zhang, H.Y., Castellanos, F.X., Milham, M.P., 2010. Toward discovery science of human brain function. PNAS 107, 4734-4739.

Biswal, B., Yetkin, F., Haughton, V., Hyde, J., 1995. Functional connectivity in the motor cortex of resting state human brain using echo-planar MRI. Magnetic Resonance in Medicine 34, 537-541.

Björnsdotter, M., Rylander, K., Wessberg, J., 2011. A Monte Carlo method for locally multivariate brain mapping. NeuroImage 56, 508-516.

Brammer, M.J., Bullmore, E.T., Simmons, A., Williams, S.C.R., Grasby, P.M., Howard, R.J., R.Woodruff, P., Rabe-Hesketh, S., 1997. Generic brain activation mapping in functional magnetic resonance imaging: A nonparametric approach. Magnetic Resonance Imaging 15, 763-770.

Bullmore, E., Long, C., Suckling, J., Fadili, J., Calvert, G., Zelaya, F., Carpenter, T., Brammer, M., 2001. Colored noise and computational inference in neurophysiological fMRI time series analysis: resampling methods in time and wavelet domains. Human Brain Mapping 12, 61-78.

Dagli, M., Ingeholm, J., Haxby, J., 1999. Localization of cardiac induced signal change in fMRI. NeuroImage 9, 407-415.

Das, S., Sen, P., 1994. Restricted canonical correlations. Linear Algebra and its Applications 210, 29-47.

Dwass, M., 1957. Modified randomization tests for nonparametric hypotheses. The Annals of Mathematical Statistics 28, 181-187.

Eklund, A., Andersson, M., Knutsson, H., 2010. Phase based volume registration using CUDA, in: IEEE International Conference on Acoustics, Speech and Signal Processing (ICASSP), 2010, pp. 658-661.

Eklund, A., Andersson, M., Knutsson, H., 2011a. Fast random permutation tests enable objective evaluation of methods for single subject fMRI analysis. International Journal of Biomedical Imaging, Article ID 627947 .

Eklund, A., Andersson, M., Knutsson, H., 2012. fMRI analysis on the GPU - possibilities and challenges. Computer Methods and Programs in Biomedicine 105, 145-161. 
Eklund, A., Friman, O., Andersson, M., Knutsson, H., 2011b. A GPU accelerated interactive interface for exploratory functional connectivity analysis of fMRI data, in: IEEE International Conference on Image Processing (ICIP), pp. 1621-1624.

Feinberg, D.A., Moeller, S., Smith, S.M., Auerbach, E., Ramanna, S., Glasser M.F., Miller, K.L., Ugurbil, K., Yacoub, E., 2010. Multiplexed echo planar imaging for sub-second whole brain FMRI and fast diffusion imaging. PloS ONE 5, e15710.

Friman, O., Borga, M., Lundberg, P., Knutsson, H., 2003. Adaptive analysis of fMRI data. NeuroImage 19, 837-845.

Friman, O., Borga, M., Lundberg, P., Knutsson, H., 2004. Detection and detrending in fMRI data analysis. NeuroImage 22, 645-655.

Friman, O., Carlsson, J., Lundberg, P., Borga, M., Knutsson, H., 2001. Detection of neural activity in functional MRI using canonical correlation analysis. Magnetic Resonance in Medicine 45, 323-330.

Friman, O., Morocz, I., Westin, C.F., 2005. Examining the whiteness of fMRI noise, in: Proceedings of the Annual Meeting of the International Society of Magnetic Resonance in Medicine (ISMRM), p. 699.

Friman, O., Westin, C.F., 2005. Resampling fMRI time series. NeuroImage 25, 859-867.

Friston, K., Josephs, O., Zarahn, E., Holmes, A., Rouquette, S., Poline, J., 2000. To smooth or not to smooth - bias and efficiency in fMRI time-series analysis. Neuroimage 12, 196-208.

Friston, K., Worsley, K., Frackowiak, R., Mazziotta, J., Evans, A., 1994. Assessing the significance of focal activations using their spatial extent. Human Brain Mapping 1, 210-220.

Gautama, T., Hulle, M.V., 2004. Optimal spatial regularization of autocorrelation estimates in fMRI analysis. NeuroImage 23, 1203-1216.

Gembris, D., Neeb, M., Gipp, M., Kugel, A., Männer, R., 2011. Correlation analysis on GPU systems using NVIDIA's CUDA. Journal of real-time image processing $6,275-280$.

Hayasaka, S., Nichols, T., 2003a. Validating cluster size inference: random field and permutation methods. NeuroImage 20, 2343-2356.

Hayasaka, S., Nichols, T., 2003b. Validation of the random field theory-based cluster size test in single-subject fMRI analyses, in: Proceedings of International Society of Magnetic Resonance in Medicine (ISMRM), p. 493.

Holmes, A., Blair, R., Watson, J., Ford, I., 1996. Nonparametric analysis of statistic images from functional mapping experiments. Journal of Cerebral Blood Flow \& Metabolism 16, 7-22.

Inselberg, A., 1985. The plane with parallel coordinates. Visual Computer 1, 69-91.

Knutsson, H., Westin, C.F., 1993. Normalized and differential convolution Methods for interpolation and filtering of incomplete and uncertain data, in: Proceedings of Computer Vision and Pattern Recognition, pp. 515-523.

Kriegeskorte, N., Goebel, R., Bandettini, P., 2006. Information-based functional brain mapping. PNAS 103, 3863-3868.

Locascio, J.J., Jennings, P.J., Moore, C.I., Corkin, S., 1997. Time series analysis in the time domain and resampling methods for studies of functional magnetic resonance brain imaging. Human Brain Mapping 5, 168-193.

Long, C., Brown, E., Triantafyllou, C., Aharon, I., Wald, L., Solo, V., 2005. Nonstationary noise estimation in functional MRI. NeuroImage 28, 890903

Lund, T.E., Madsen, K.H., Sidaros, K., Luo, W.L., Nichols, T.E., 2006. Nonwhite noise in fMRI: Does modelling have an impact? NeuroImage 29, 54-66.

Luo, H., Puthusserypady, S., 2007. fMRI data analysis with nonstationary noise models: A bayesian approach. IEEE Transactions on Biomedical Engineering 54, 1621-1630.

Martino, F.D., Valente, G., Staeren, N., Ashburner, J., Goebel, R., Formisano, E., 2008. Combining multivariate voxel selection and support vector machines for mapping and classification of fMRI spatial patterns. NeuroImage $43,44-58$.

Milosavljevic, M.M., Veinovic, M.D., Kovacevic, B.D., 1995. Estimation of nonstationary AR model using the weighted recursive least square algorithm, in: IEEE International Conference on Acoustics, Speech and Signal Processing (ICASSP), 1995, pp. 1432-1435.

Mitra, P.P., Pesaran, B., 1999. Analysis of dynamic brain imaging data. Biophysical Journal Volume 76, 691-708.

Mourao-Miranda, J., Bokde, A.L., Born, C., Hampel, H., Stetter, M., 2005. Classifying brain states and determining the discriminating activation patterns: Support vector machine on functional MRI data. NeuroImage 28 ,
980-995.

Nandy, R., Cordes, D., 2003. A novel nonparametric approach to canonical correlation analysis with applications to low CNR functional MRI data. Magnetic Resonance in Medicine 49, 1152-1162.

Nandy, R., Cordes, D., 2007. A semi-parametric approach to estimate the family-wise error rate in fMRI using resting-state data. NeuroImage 34, 1562-1576.

Nichols, T.E., Hayasaka, S., 2003. Controlling the familywise error rate in functional neuroimaging: a comparative review. Statistical Methods in Medical Research 12, 419-446.

Nichols, T.E., Holmes, A.P., 2001. Nonparametric permutation tests for functional neuroimaging: A primer with examples. Human Brain Mapping 15, $1-25$.

Norman, K.A., Polyn, S.M., Detre, G.J., Haxby, J.V., 2006. Beyond mindreading: multi-voxel pattern analysis of fMRI data. Trends in Cognitive Sciences 10, 424-430.

Poline, J., Worsley, K., Evans, A., Friston, K., 1997. Combining spatial extent and peak intensity to test for activations in functional imaging. NeuroImage 5, 83-96.

Purdon, P.L., Weisskoff, R.M., 1998. Effect of temporal autocorrelation due to physiological noise and stimulus paradigm on voxel-level false-positive rates in fMRI. Human Brain Mapping 6, 239-249.

Siegel, S., 1957. Nonparametric statistics. The American Statistician 11, 13 19.

Smith, A., Lewis, B., Ruttimann, U., Ye, F., Sinnwell, T., Yang, Y., Duyn, J., Frank, J., 1999. Investigation of low frequency drift in fMRI signal. NeuroImage 9, 526-533

Smith, A.T., Singh, K.D., Balsters, J.H., 2007. A comment on the severity of the effects of non-white noise in fMRI time-series. NeuroImage 36, 282-288.

Tillikainen, L., Salli, E., Korvenoja, A., Aronen, H., 2006. A cluster mass permutation test with contextual enhancement for fMRI activation detection. Neuroimage 32, 654-664.

Woolrich, M.W., Ripley, B.D., Brady, M., Smith, S.M., 2001. Temporal autocorrelation in univariate linear modeling of FMRI data. NeuroImage 14, 1370-1386.

Worsley, K., Liao, C., Aston, J., Petre, V., Duncan, G., Morales, F., Evans, A., 2002. A general statistics analysis for fMRI data. NeuroImage 15, 1-15.

Zarahn, E., Aguirre, G., D’Esposito, M., 1997. Empirical analyses of BOLD fMRI statistics I. Spatially unsmoothed data collected under null-hypothesis conditions. NeuroImage 5, 179-197. 